\title{
Flow structure and shear generation in 3D SOL of circular tokamak plasma in Aditya
}

\author{
Bibhu Prasad Sahoo, ${ }^{1}$ Devendra Sharma, ${ }^{1}$ Ratneshwar Jha, ${ }^{1}$ and Yühe Feng ${ }^{2}$ \\ ${ }^{1}$ Institute for Plasma Research, Bhat, Gandhinagar, India, 382428 \\ ${ }^{2}$ Max-Planck-Institut für Plasmaphysik, Wendelsteinstr. 1, Greifswald, D-17491, Germany
}

(Dated: April 12, 2015)

\begin{abstract}
Presence of a poloidal ring limiter in the tokamak Aditya which has a circular plasma presents an interesting Scrape-off Layer (SOL) plasma configuration where small to large scale spatial variations of plasma parameters are expected. Flow shear in the Aditya SOL are routine observations and their strong correlation with the experimentally measured edge fluctuations in floating potential and SOL currents/drifts are thoroughly investigated in the recent past. The first implementation of 3D SOL plasma transport simulation code EMC3-EIRENE allows possibility to investigate the SOL complexity and associated short and long scale spatial patterns in the flow and plasma transport, with its impact on the confinement properties of the plasma in edge region. A detailed investigation of first results from the 3D plasma transport simulations shows interesting 3D flow patterns and existence of resulting parallel and radial flow shear in the device SOL. In the present work the origin of these complex flow patterns is studied and their correlations with observed behavior of the flows in device is discussed.
\end{abstract}

\section{INTRODUCTION}

Plasma transport in the Scrape-off Layer (SOL) of a circular cross-section limiter plasma is simpler in its analytic structure. In the present day scenario with increasing complexity of the plasma wall interaction region in the fusion devices, the analysis of plasma transport in the devices with such simpler SOL configurations can provide valuable inputs for the validation and determining the applicability of analytic transport models, particularly those underlying advanced computer simulations. For the latter, data from the simpler configurations may allow an effective benchmark with the experimental observation which is essential for refining the SOL transport models applied for computations in many $2 \mathrm{D}$ and 3D SOL simulation tools [1-4]. The degree of complexity of the turbulent plasma transport in SOL in comparison to that in the core is explored in many experiments where SOL plasma flows are measured [5-8]. This variation in the transport characteristics can be decisive to the confinement regimes as well as over all plasma stability since differential transport characteristics across the Last Closed Flux Surface (LCFS) [9-11] can be key to a desired pedestal structure or transport barrier [12-14], impacting directly the processes like L-H transition $[15,16]$. The recent observations and computational analysis of circular plasma of tokamak Aditya showed a complex structure of flows in the limiter generated SOL of the device [17-19]. It was shown that the flow structure in case of a toroidally localized poloidal limiter can be sufficiently complicated even in the cases of a circular limiter generated SOL of the tokamak, thereby disallowing an easy interpretation of the flow measurements done using probes. In such cases a combination of experimental and computational means can be particularly effective in characterizing the SOL flow structure, given that the two approaches can complement each other's capacity. In the SOL transport of tokamak Aditya studied here, there are signatures that the spatial complexity of flow structures observed in the present steady-state EMC3EIRENE simulations might be capable of strongly perturbing the drifts and flows that are routinely measurable in the experiments but excluded from the simulations for the simplicity. Evolution of such drifts and flows modulated by the steady-state structures is a plausible source of the spatial and temporal fluctuations in the edge region. Such activity, besides being regularly observed, influences the critical factors like local particle and heat transport coefficients, determining, in turn, the stability of the overall equilibrium.

In Aditya, which has major radius $75 \mathrm{~cm}$, minor radius $25 \mathrm{~cm}$ and a maximum toroidal magnetic field of $\sim 0.72 \mathrm{~T}$ at the magnetic axis, the circular plasma is limited by a poloidal ring limiter localized at one toroidal location. The successful plasma operations on Aditya over last few decades have covered many aspects of edge plasma physics $[20,21]$ in its simple circular core plasma which is interfaced with a purely $3 \mathrm{D}$ SOL since the variations are expected in all, poloidal, radial and toroidal directions in presence of the poloidal limiter. The recent experiments involving flow measurements in Aditya SOL [17, 18] have been focused on studying the interaction and control of the SOL and edge plasma by means of external control mechanisms, like a gas puff. Many interesting effects of these external mechanisms, such as suppression of floating potential fluctuation, were also noted and efforts were made to establish their correlations with the global variation in the parameters like, floating potential, plasma density and the Reynolds stress [18]. In a more recent set of experiments, special attempts were also made to recover the transport and drift driven components from the net flow measured in the SOL using Mach probes installed at various toroidal and poloidal locations by affecting a magnetic field reversal [22, 23].

Behavior of plasma transport, flows, drifts and their associated fluctuations are studied routinely in the edge and SOL region of tokamak Aditya by analyzing the data from Mach probes and Langmuir probes [17, 18, 22]. 
Many useful conclusions were drawn based on the probe measurements which were employed to estimate flows that mainly include the components along the toroidal and poloidal directions. The probe measurements however remain highly local and limited in their capacity to provide information about the global spatial variations because of limited number of probes on the device. The toroidal Mach number, for example, was observed to increase with radial distance in the SOL and decrease with increasing density [17]. An interpretation of this was provided in terms of flows that are global in nature since they originate because of the following two factors: (i) particle drift effects that are identified to develop at longer scale lengths, corresponding to that of a mean electric field (e.g, $E \times B$ and Pfirsch-Schluter flows that develop due to vertical charge separation at the minor radius) and (ii) the pressure difference driven flows that develop due to the plasma source, in the form of diffusion across the LCFS, over the SOL length, largely comparable to maximum connection length, $L_{c}$. Here $L_{c}$ is typically the separation along the magnetic field lines between the strike points at the two opposite faces of the limiter. In the present set up, for example, the toroidal Mach number (that shows a radial variation) is measured at two spatial locations on the device while floating potential and density measurements were done at three locations, by combining the data derived both from the Mach probes and the Langmuir probes [18]. The absence of measurements from rest of the volume of 3D SOL generated by a poloidal limiter was complemented in the accompanying analysis by making reasonable assumption about the global structure of the variation in quantities that are measured only locally. While this approach has been successful in analyzing many aspects of the observations, the underlying assumptions need to be substantiated and improved further in order to cover the areas where the ambiguity still exists. An improved estimate requires data which is global in nature and allows to correlate the local behavior of the measurements to the global features of the parameter variations modeled by approximate means, e.g., using a schematic slab model as done in the recent data analysis [18, 22].

In a simple slab model used in order to interpret the data from a Mach probe and Langmuir probe measurements, the effects on the plasma parameters at the measurement locations is modeled by estimating the effects of various nonlocal factors. Some of these factors, for example, a localized gas puff and the sink action by a limiter, are strongly localized at a far off location from the probes along the magnetic field in the toroidal direction such that their effects can be estimated only to a limited accuracy. In absence of the sufficient spatial continuity in the data from the probe measurements to these localized source of controlling factors, the 3D simulations are seen as the best tool for estimating the missing information and completing or refining the present global picture based on qualitative assumptions. The first 3D simulations of the plasma transport in the Aditya SOL using the EMC3-EIRENE code [3, 4] have already indicated some interesting features of the plasma parameters and the plasma flow profiles in the device SOL that comprises of various regions of very small to very long connection lengths [19]. Over the intermediate period we have performed a more detailed simulation study and analysis of the resulting data, including a first comparison and correlation of this data to the actual measurements on the device.

In previously reported initial studies, interesting 3D flow patterns were predicted in the SOL region emerging mainly from the sharp changes in the connection lengths in the SOL region [19]. The associated $3 \mathrm{D}$ variation in the SOL plasma parameters resulting from these stationary structures are further analyzed and obtained to be quasi-periodic in the poloidal direction. Since the structures are associated with the $q$-profiles and therefore are static in nature over the flat-top duration, their average effect on the drift and flow profiles in the SOL and edge region are expected to be however small but non-zero. In the present analysis this effect is estimated by obtaining, from the 3D simulation data, the residual poloidal and radial density gradients and the resulting diamagnetic drifts. Considering such drifts to be the source of free energy for potential fluctuations and large scale activities, an estimation of their effects indicates a possibility of additional vorticity generation in the SOL resulting from a self-terminating diamagnetic drift [24] in the region of flow structures that introduce a static quasiperiodic poloidal variation in the SOL plasma parameters. The resulting vorticity in the routine SOL flows is indicative of the capacity of the flow perturbations to influence the degree of desired differential anomalous diffusivity across the LCFS (the boundary separating the regions of closed and open field line regions) which is one of the parameters determining the radial field or the strength of an edge transport barrier, given a strong correlation of a jump in the $D_{\perp}$ across the LCFS to the plasma confinement regime.

For the cases simulated in the present analysis, using the range of the parameter values consistent with the experimental conditions, we have discussed our simulation output with respect to the existing observations on the device. Areas are indicated where the simulated data can complement the experimental measurements and possibly motivate the organization of the future experiments on the device. The present implementation of EMC3-EIRENE to Aditya SOL flow studies involves adapting a plasma equilibrium and generation of an optimum 3D mesh with variable resolution that allows sampling the variation in plasma parameters in the region of large gradients with enough accuracy as well as provides good statistical accuracy and computational efficiency with coarser mesh in the region where gradients are relatively small. In the present paper, the structure of the ring-limiter generated SOL is discussed in the Sec. II along with the procedure of the application of the code to Aditya SOL, followed by the description of 
(a)

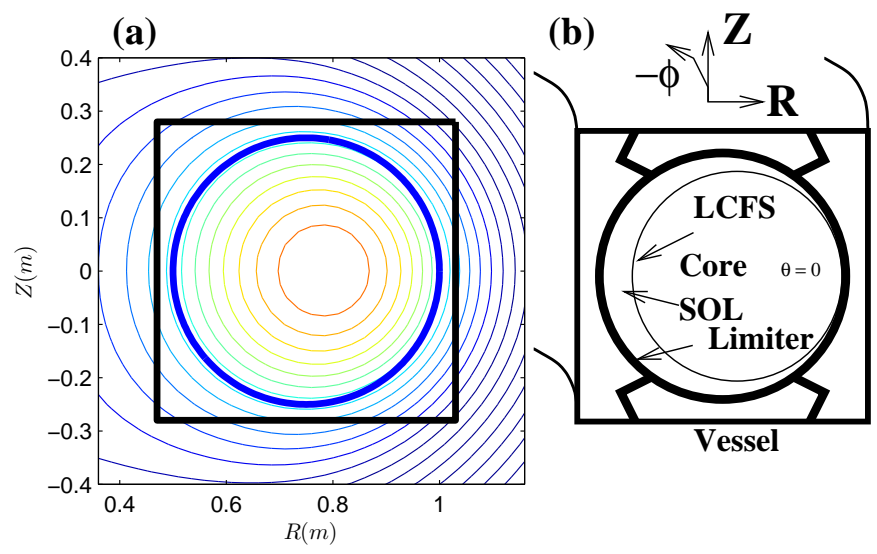

FIG. 1: (a) Contours of flux surfaces obtained from the magneto-hydrodynamic equilibrium calculations with the inner surface of the limiter represented by the thick circle and cross-section of the vessel represented by thick square. (b) Schematic of the vessel intersection at the location of the left face of the limiter $\phi=0^{\circ}$ facing the direction $-\hat{\phi}$, where $\hat{\phi}$ is the direction clockwise along the torus as seen from the top.

the plasma-neutral transport model adopted in the code EMC3-EIRENE in Sec. III and analysis of the simulation results in Sec. IV. The computational results are analyzed with respect to the existing probe measurements in the device SOL in Sec. V, and the effects of observed flow structures are estimated in Sec. VI. Summary and conclusions are presented in Sec. VII.

\section{COMPLEX SOL IN ADITYA AND THE EMC3-EIRENE COMPUTATION GRID}

The schematic slab model used in the existing interpretation of the probe data relies upon modeling the SOL region as a perfect $3 \mathrm{D}$ slab with two of its opposite sides representing the two faces of the limiter, such that the entire geometric volume between these faces is approximated as a region of open magnetic field lines. In the present treatment this model is upgraded by deriving the topology of the magnetic field lines in the plasma volume from the magnetic flux data obtained from the numerical computation of an exact magneto-hydrodynamic (MHD) equilibrium for Aditya [25] computed using standard numerical procedures [26]. The modified approach is based on the mapping of the limiter geometry to a slab while considering the relative curvature of the equilibrium magnetic surfaces and the poloidal limiter. This approach produces many important improvements in the understanding the complexity of the structure of the SOL of Aditya which is produced by a toroidally localized poloidal continuous limiter. The first modification in the slab model comes from accounting for the Shafranov shift produced in the magnetic flux surfaces as a result of the toroidal version of the fundamental magnetohydrodynamic equilibrium, $\mathbf{j} \times \mathbf{B}=-\nabla p$, where $\mathbf{j}$ is the

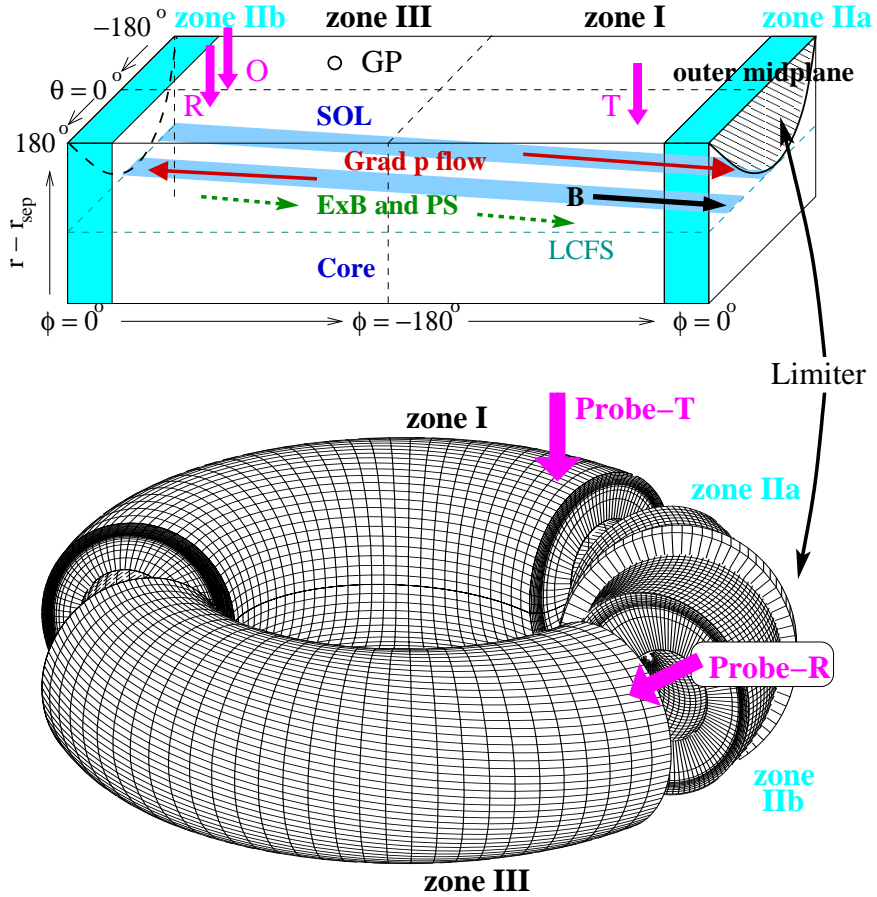

FIG. 2: Schematic of the improved slab model to interpret the probe data (top) and a corresponding realistic 3D transport simulation grid for EMC3-EIRENE simulation (bottom) derived from the Aditya MHD equilibrium.

toroidal plasma current density, $\mathbf{B}$ is the total magnetic field and $p$ is the plasma pressure. As evident from the cross-sectional view of magnetic flux surfaces plotted in Fig. 1 (showing also the limiter and the vessel), this shift towards the Low Field Side (LFS) in the plasma center and therefore in the nearly circular magnetic surfaces, results in the poloidal limiter intersecting the magnetic surfaces having larger poloidal curvature than that of the ring-shaped limiter, as described schematically in Fig. 2.

It is clear from the transformation of toroidal magnetic flux surfaces to a 3D slab as shown in Fig. 2(top) that, once the set of nested equilibrium magnetic flux surfaces is represented by a set of stacked $2 \mathrm{D}$ planes such that they can be accommodated, horizontally, in a 3D slab, the limiter ring that has a curvature different than the flux surfaces can clearly be represented only by a convex structure intersecting the set of horizontal planes inside the slab with larger radius. Note that the first horizontal plane intersecting the limiter, specifically the plane intersecting the tip of the convex limiter structure, represents the LCFS. As a result of this curved limiter image in the slab, the SOL region, which is essentially the volume occupied by the bunch of field lines connecting both the faces of the limiter, must also be a region of finite curvature and not perfectly bounded by the LCFS since the latter is a flat surface in the transformed slab geometry. Considering the narrow area of the limiter wetted by the plasma at the outboard midplane [17], when 

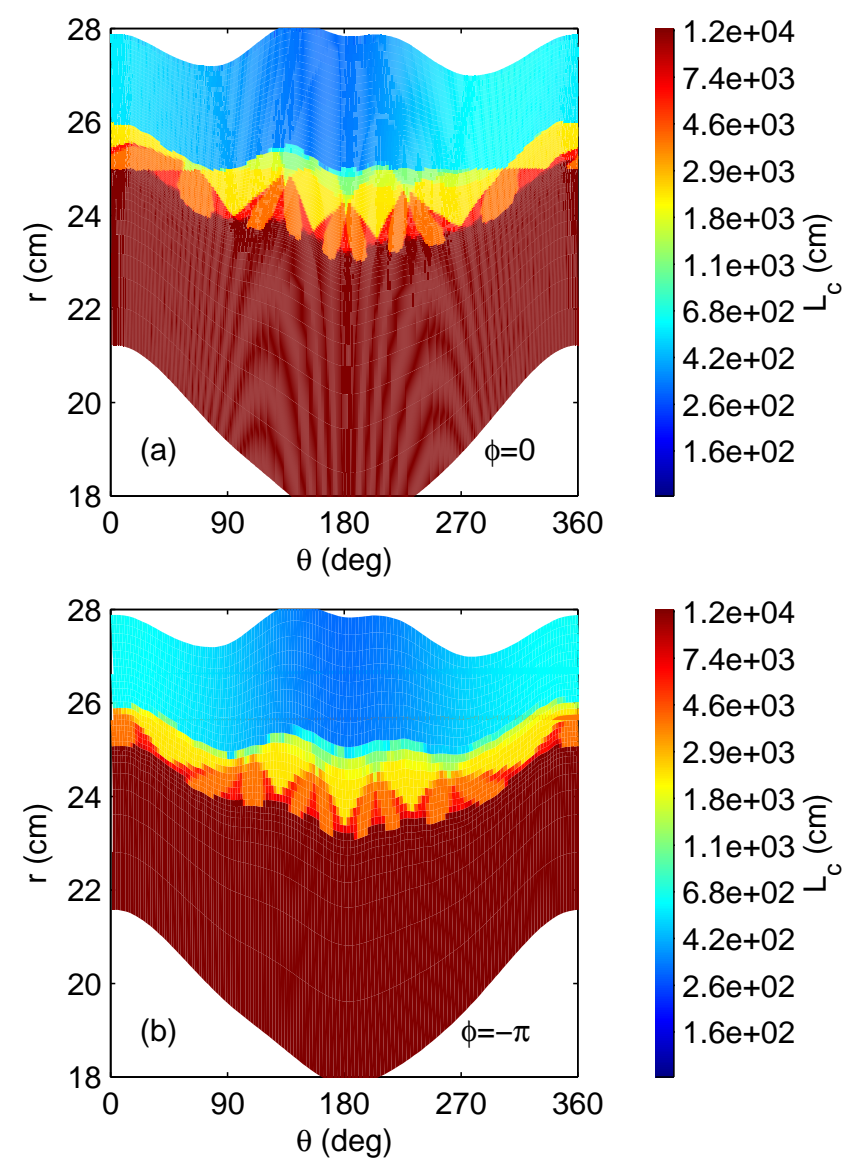

FIG. 3: The radial-poloidal distribution of the connection length at (a) limiter face $\phi=0^{\circ}$ and at the location diagonally opposite to the limiter face $\phi=-180^{\circ}$. The angles $\theta=0^{\circ}(\equiv$ $360^{\circ}$ ) and $180^{\circ}$ correspond to the outboard and inboard sides, respectively.

the image of this wetted area is followed along the magnetic field lines on the LCFS, it traces a narrow band of field lines on the LCFS. This band, depending on the $q$ value of the operation, is likely to intersect the opposite face of the limiter only following multiple toroidal turns along the LCFS (indicated schematically in Fig. 2(top) by the bands drawn originating from the limiter tips). The LCFS thus emerges as a surface with an alternating combination of narrow zones of open and closed field lines depending up on the $q$ value of the operation. This can lead to a significant impact on various aspects of operations, especially in the steady-state conditions involving higher power load as considered in the earlier EMC3EIRENE studies [27]. Note that depending up on LCFS being an irrational or rational surface, the $q$ value determines the contrast in the connection lengths in these alternating zones on the LCFS. For the poloidal limiter in Aditya (with $q=r B_{t} / R B_{p}=3.3$ maximum at the SOL boundary, $r=25 \mathrm{~cm}$ ), the above picture must be correct for all the magnetic surfaces beyond the LCFS until one reaches a radius where the surfaces begin to intersect the entire poloidal extension of the limiter, represented by the surface on the top face of slab in the schematic Fig. 2(top). The connection length distribution on the radial-poloidal plane computed using EMC3EIRENE shows this variation as drawn in Fig. 3(a) and (b) at the limiter face $\phi=0^{\circ}$ and at the toroidal location diagonally opposite to the limiter location $\phi=-180^{\circ}$, respectively. It is additionally seen from Fig.3(a) that, while the flux surface in the core region have a single radius of curvature, different from that of the circular limiter (represented on this plane by the straight line $r=25 \mathrm{~cm}$ ), the outer surfaces have an increasing variation in their radius of curvature in the device, making some of them intersect the circular limiter at multiple $\theta$. The SOL region in the device is therefore expected to be strongly filamented where the filaments have variations in their dimensions along all the three, toroidal, radial and poloidal directions. Considering strong parallel flows in the SOL, an associated complexity in the parallel and cross-field (radial and poloidal) plasma transport can thus be anticipated in the filamented SOL region beyond the LCFS. It can be easily realized that the variation in the poloidal dimension of the filaments would be far more limited in the case of a discontinuous poloidal limiter [28] as compared to the present continuous case where the successive magnetic surfaces beyond LCFS intersect increasingly larger poloidal extension of the ring limiter. This effectively results in increasing width of the bands on corresponding flux surfaces similar to those drawn on the LCFS in Fig. 2(top).

The methodology in the advanced computational investigation of the SOL transport presented in the present analysis further extends to utilizing the power of $3 \mathrm{D}$ plasma transport simulation capacity of the code EMC3EIRENE and characterizing the effects of this complex SOL configuration on the plasma transport in the Aditya SOL. As already mentioned, employing EMC3-EIRENE simulations allows a large degree of refinement in the analysis of limited probe measurements that are routinely interpreted using only a schematic slab model. This is more obvious from the fact that the EMC3-EIRENE essentially uses a rather accurate slab model based on the magnetic flux surface data derived from the equilibrium computations. In case of Aditya SOL it additionally completes the global picture mostly assumed in the existing analysis of the measurements, by producing quantitative transport data in the form of 3D solutions of SOL plasma transport model implemented in EMC3-EIRENE code combination. The simulation procedure begins by constructing an optimized 3D mesh to adequately represent and sample the complexities of the SOL and properly resolve the effects it produces in the transport of plasma particles, momentum and energy.

The experimental investigations on the device Aditya are limited to a few locations largely because of the few probes located at large enough toroidal distance from the each of two opposite sides of the limiter and are also separated considerably from each other in poloidal and 
toroidal dimensions. The conclusions about the local as well as global gradients based on probe measurements have therefore been largely approximate as any information of the global distribution of the plasma parameters must come only from the qualitative assumptions. These qualitative assumptions in the present computational analysis are used rather for optimizing the 3D mesh that must be constructed carefully for producing more accurate quantitative estimates from the simulations.

Considering, for example, that the poloidal limiter has a small wetted area, the indications are that strong localized gradients must develop in zones close to the narrow strike point which is sufficiently localized both in poloidal and radial dimensions on the limiter. The 3D mesh for EMC3-EIRENE computations therefore must be constructed with enough resolution in such regions keeping this strong 3D variation in the view. Depending on the requirements of sufficient spatial resolution in particular regions the mesh has also been subdivided in three toroidal zone with varying resolution of both geometrical and physical cell sizes, as required for both, solving particle transport and scoring the Monte-Carlo particles in order to build up the statistical averages. The view of a typical set of toroidal-poloidal surfaces forming the torus is presented in Fig. 2(bottom) showing the varying resolution of the geometrical cells. The presence of limiter is indicated in the Fig. 2 at $\phi=0^{\circ}$ by drawing the ring like structure intersecting the outer surface. The physical cells are constructed by grouping further a finite number of geometrical cells on the surfaces, together, for an optimum quality of statistical averages and their gradients $[3,4]$. This subdivision of the grid in various toroidal zones and smaller cells, even in the region where no large toroidal gradients are expected, is required also for an additional reason, particularly, since the 'reversible fieldline mapping' technique [29] adopted in EMC3 requires convex helical cells in order to avoid mutual intersection of the cell faces which is possible only for a limited field line length along the magnetic field lines. The second requirement, as discussed above, comes from the recycling region around the limiter that needs finer resolution to resolve larger gradients. As presented in Fig. 2 among the three zones covering the entire volume of the device SOL, the zone-I and zone-III are two symmetric zones with moderate resolution extending from the toroidal location $\phi= \pm 180^{\circ}$ to $\phi= \pm 30^{\circ}$, respectively, while higher resolution is used in a smaller zone-II that extends from $\phi=-30^{\circ}$ to $30^{\circ}$ and in which the limiter is located. Following the conventional mode of operations in the device, the left face (or the ion collecting face, facing the direction $-\hat{\phi}$ ) of the limiter is located at the center of the zone-II of the mesh, i.e., at the toroidal location $\phi=0^{\circ}$.

Using the simplifications which the circular crosssection of the Aditya plasma allows in comparison to the case of a diverted plasma [30], the 3D mesh is constructed with a rather simple, single surface (i.e., SOL-core interface) extending the entire poloidal range and subdivision is only required along the toroidal direction where the magnetic configuration is divided into three toroidal simulation zones for different reasons. This simplicity of the device and the computational procedure enables the simulation results to be free from additional complexities in specially shaped plasmas that are used from the view points of better stability and control. This brings us to pointing out that one of the notable advantages of the implementation of the code to the simple circular plasma in a working device like Aditya is the scope of a relatively simpler benchmark of the computational results with the experimental profiles which are expected to be more analytic in nature considering the inherent symmetries of the plasma in the device. Since the experimental measurements on the device contain most of the first and higher order effects, that are absent from the simulation model presently in use (e.g., drifts and currents), the inclusion of these effects in the simulation model in the near future may possibly benefit from the comparison of the experimental measurements on the device with the present simulation results that exclude the higher order effects generally expected in shaped plasmas.

\section{PLASMA-NEUTRAL TRANSPORT MODEL}

Originally applied to simulate the edge plasma, the neutral particle and the impurity transport in full 3D geometry the code EMC3-EIRENE was developed and extensively used for simulating routine plasma operations in intrinsically three dimensional stellarator W7-AS and other helical devices [31-33]. Over the last few years, however, the code also found applications in the tokamak community [19,30,34-38], in particular, tokamaks with limiter plasmas where either MP (Magnetic Perturbation) coils or other elements break the 2D toroidal symmetry $[19,30,35,36,38]$. The version of EMC3 code employed for the present computations implements a stochastic Monte-Carlo procedure to solve the collisional Braginskii fluid equations and compute transport of the plasma species. The neutral transport is simulated by the EIRENE code which implements a complete kinetic model for the neutral particles, whereas the parallel transport description is purely classical in EMC3, anomalous cross-field transport coefficients $D, \eta$ and $\chi$ are usually assumed as free parameters in the code. Although a Monte-Carlo principle is applied to solve the equations numerically, the code implements a fluid model to describe the plasma transport including all the approximations of the fluid picture. The present version of EMC3 however includes a less comprehensive set of atomic processes (volumetric recombination is excluded for example) and the drifts and flows related to vertical charge separation are not taken into account. In the view that the objective of the present analysis is rather to identifying complex but symmetric structure of the transport driven flows in order to separate the additional features observable in the actual flow measurements, the existing level of simplification of the computational pro- 
cedure, which may still allow one to achieve this limited objective, is tolerated.

On a 3D mesh, constructed following magnetic flux surface of arbitrary geometry, the set of steady state fluid equations solved by the adopted version of the EMC3EIRENE code includes the balance equations for, density $n$, parallel momentum $m v_{\|}$and energies of electrons and ions, $T_{e}$ and $T_{i}$, respectively, given as,

$$
\begin{array}{r}
\nabla_{\|} \cdot n \vec{v}_{\|}+\nabla_{\perp} \cdot\left(-D \nabla_{\perp} n\right)=S_{p} \\
\nabla_{\|} \cdot\left(m n \vec{v}_{\|} \vec{v}_{\|}-\eta_{\|} \nabla_{\|} v_{\|}\right) \\
+\nabla_{\perp} \cdot\left(-m v_{\|} D \nabla_{\perp} n-\eta_{\perp} \nabla_{\perp} v_{\|}\right) \\
=-\nabla_{\|} p+S_{m} \\
+\nabla_{\perp} \cdot\left(-\frac{5}{2} T_{i} D \nabla_{\perp} n-\chi_{i} n \nabla_{\perp} T_{i}\right) \\
=K\left(T_{e}-T_{i}\right)+S_{e i} \\
\left(\frac{5}{2} n T_{e} \vec{v}_{\|}-\kappa_{i} \nabla_{\|} T_{i}\right) \\
\left.+\nabla_{\perp} T_{e}\right) \\
\left(-\frac{5}{2} T_{e} D \nabla_{\perp} n-\chi_{e} n \nabla_{\perp} T_{e}\right) \\
=K\left(T_{i}-T_{e}\right)+S_{e e}
\end{array}
$$

where the subscripts $\|$ and $\perp$ indicate vectors along and across the magnetic field directions with $\mathrm{p}=\mathrm{n}\left(T_{e}+T_{i}\right.$ ). Effects, along the direction parallel to the magnetic field, of the transport coefficients $\eta, \kappa_{e}, \kappa_{i}$ are as prescribed by the classical transport theory by Braginskii, while anomalous cross-field transport is taken into account by free model parameters $D, \chi_{e}, \chi_{i}$ and fixed viscosity $\eta_{\perp}=m_{i} n D$. Interactions with neutral particles are taken into account by the sources of particle, momentum and energy associated with neutrals, $S_{p}, S_{m}, S_{e e}$ and $S_{e i}$, respectively, which are calculated by coupling the EMC3 plasma transport computations with the neutral transport code EIRENE. An iterative procedure is applied to obtain a self-consistent solution for all plasma and neutral particle parameters.

The SOL plasma transport simulations in EMC3EIRENE uses boundary conditions in the form of density value at the core-SOL interface and the plasma flow velocity at the target boundaries where the field lines are incident on the target. The parallel flow velocities of the species are accordingly set to be the ion acoustic speed of the species $\mathrm{H}+$ for the present simulations. The parallel transport coefficients have their classical values in the underlying collisional fluid model of the plasma while the particle and heat diffusion across the magnetic field are anomalous and are empirically set to be $D=1 \mathrm{~m}^{2} / \mathrm{s}$ and $\chi=3 D$ for the present computations, and effect of their variations are excluded from the present study for simplicity. An effective radial decay length is used on the radial boundary of the plasma other than targets.

The typical plasma discharges that are considered for simulations in this study are selected from the examples where the flow and potential probe measurements in the SOL are already reported in the earlier studies $[17,18,22]$. For example, Mach number measurements were made in the discharges with plasma current, $I_{p} \sim 25$ $\mathrm{kA}$, plasma duration $\sim 25 \mathrm{~ms}$, core electron temperature $\sim 100 \mathrm{eV}$, center chord averaged plasma density $5 \times 10^{12}$ $\mathrm{cm}^{-3}$, SOL plasma density $5 \times 10^{11} \mathrm{~cm}^{-3}$ using hydrogen as working gas [17]. The connection lengths in the SOL region measure a few tens of meters $(\gtrsim 50 \mathrm{~m})$ as compared to the ion-ion mean-free-path $(\sim 8-50 \mathrm{~m}$, considering $T_{e} \sim 20-50 \mathrm{eV}$ ), allowing the application of the collisional simulation model. Considering the above parameters, particularly the loop voltage $V_{L} \sim 2-3 \mathrm{~V}$ in combination with the plasma current $I_{p} \sim 25 \mathrm{kA}$, an input power about $60-70 \mathrm{~kW}$ is estimated. Since the total power across the core-SOL interface, $\mathrm{P}$, is a user input for the EMC3-EIRENE computations which, in turn, determines the total recycling flux and the species temperature distribution in the simulation zone, a fixed value, $P=60 \mathrm{~kW}$, is used for the cases simulated in the present computations along with the core-SOL interface density value $5 \times 10^{11} \mathrm{~cm}^{-3}$. This net power input apart from determining the scale of the overall 3-dimensional electron and ion temperature profiles, controls the ionization profiles via various atomic and molecular processes modeled in the Monte-Carlo neutral code EIRENE which is coupled with the plasma computations done by the code EMC3. In the vacuum region beyond the SOL boundary a coarser mesh is used to sample the neutral particle distributions determined by EIRENE. Computations are performed to analyze the resulting equilibrium profiles of plasma parameters, namely, plasma density, electron and ion temperatures and plasma flow velocities with a set of prescribed input parameters, i.e., the density at the core-SOL interface and the input power.

\section{TRANSPORT IN THE FILAMENTED SOL}

The code EMC3-EIRENE has successfully been applied recently to many tokamak SOL where the origin of $3 \mathrm{D}$ effects relates to stochastic magnetic fields which is used in order to effectively modify and control the perpendicular transport characteristics [30, 35]. The resulting magnetic field perturbations in such intrinsically $3 \mathrm{D}$ setup is capable of causing strong influence on the radial gradients of the equilibrium plasma parameters as simulated by $3 \mathrm{D}$ computations. The mechanism essentially works by breaking the toroidal symmetry of the tokamak that affected the plasma flow in the edge. The mechanism of the filamentation of the SOL region described above can potentially result into an analogous setup to result in the local flow perturbations that interact with the zero order flows or drifts in the SOL, modifying them following interaction between the two flow structures with two different scales. The local flow perturbations are seen to be associated with a combination of spatial zones having 

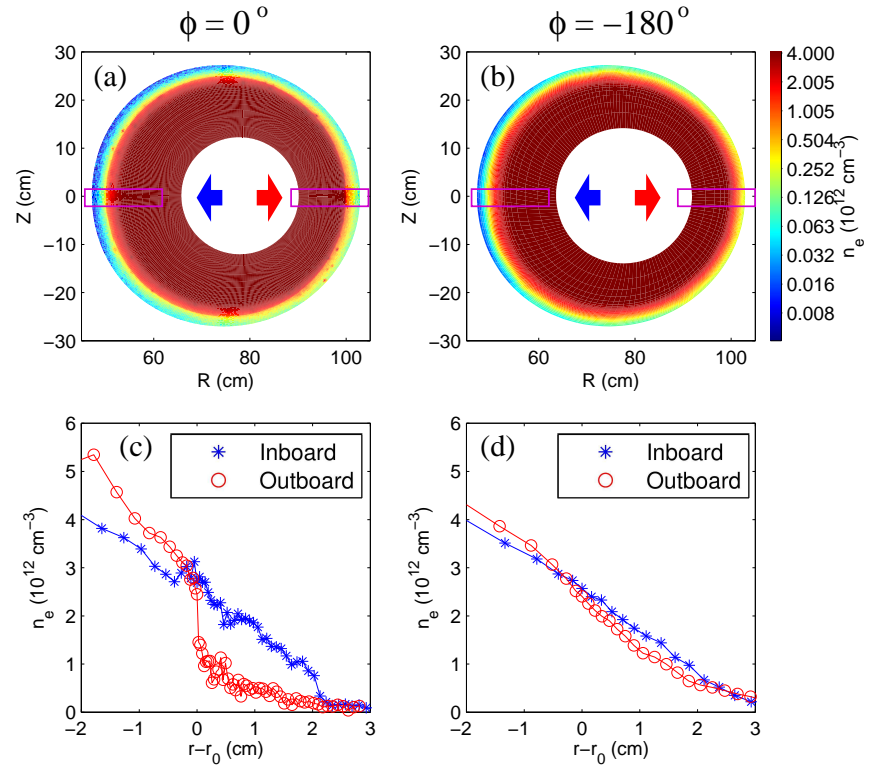

FIG. 4: 2D Radial-poloidal cross-section of the simulated 3D density distribution at the toroidal locations (a) $\phi=0^{\circ}$ or the limiter location and (b) $\phi=-180^{\circ}$ or the diametrically location opposite to the limiter plane. Radial profiles of the density on inboard $\left(^{*}\right)$ and outboard (o) sides at toroidal locations (c) $\phi=0^{\circ}$ and (d) $\phi=-180^{\circ}$.

a considerably strong variation in the connection lengths in the SOL, which can be compared to the spatial distribution of the connection lengths associated with the region of resonant magnetic perturbations. It is therefore of general interest to analyze the SOL plasma transport characteristics in presence of such complex distribution of connection-lengths in a relatively simpler Aditya SOL configuration.

In case of a toroidally localized poloidal limiter, as in Aditya, it must be most reasonable to examine the simulations results at the location close to the limiter and farthest from it, i.e., $\phi=-180^{\circ}$, in order to find out what degree of contrast exists between these two extremes since these two toroidal locations correspond to the recycling zone and the stagnation point, respectively, and therefore the plasma conditions associated with them largely determine the global structure of the zero order pressure driven flows. Considering, for example, the power and particle sources from the core to be approximately uniformly along the toroidal direction, the energy and particle fluxes build up beginning from the stagnation point and moving downstream along the toroidal axis up to the target location from either of the sides (clockwise or counter clockwise) and the gradients in the plasma parameters are expected in the entire toroidal extension of the SOL. The gradients are generally expected to be sharper in the region of plasma close to the limiter, even for the uniform sources from the core, primarily because the limiter at a single toroidal location is effectively a strong localized sink of the plasma in
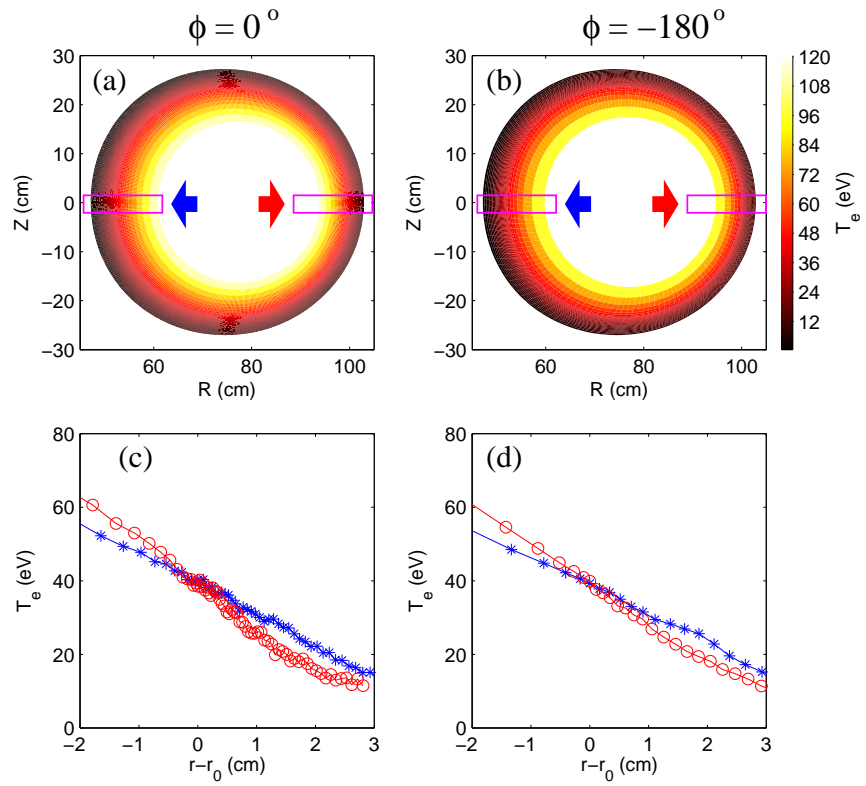

FIG. 5: 2D radial-poloidal cross-section of the simulated 3D electron temperature distribution at the toroidal locations (a) $\phi=0^{\circ}$ or the limiter location and (b) $\phi=-180^{\circ}$ or the diametrically location opposite to the limiter plane. Radial profiles of the electron temperature on inboard $(*)$ and outboard (o) sides at toroidal locations (c) $\phi=0^{\circ}$ and (d) $\phi=-180^{\circ}$.

the SOL. In our analysis of 3D results from our simulations we therefore present and analyze plasma parameters at these two locations as in Fig. 4 and beyond, and in the following discussion. However, in our effort to establish correlations between the simulations results and the probe measurements from the device SOL, we additionally focus and present the data also from two additional important toroidal locations which correspond to the locations of probes on the device which are indicated and described in the Sec. II with the help of Fig. 2.

The plasma density and temperature distributions are drawn from the $3 \mathrm{D}$ simulation data and presented in Fig. 4 for the plasma cross-section at the toroidal location $\phi=0^{\circ}$ and $-180^{\circ}$, that correspond to the radial-poloidal plane formed by the cells just before the left face (facing $-\hat{\phi}$ direction) of the limiter and those forming the plane diametrically opposite to the limiter, respectively. The density distribution at $\phi=0^{\circ}$ plotted in Fig. 4(a) shows that the density in the outboard side drops very sharply to smaller values $\left(<10^{12} \mathrm{~cm}^{-3}\right)$ at the radius corresponding to the LCFS, $r=r_{0}$, while at $\phi=-180^{\circ}$ the density at the outboard side has a gradual fall across the LCFS location. This drop is elaborated in the $2 \mathrm{D}$ plot profiles of the density in Fig. 4(c) where radial profile of the outboard density is plotted with circles at limiter location $\phi=0^{\circ}$. Considering a source of plasma from the core into the SOL, predominantly from the low field (outboard) side, the density builds up at upstream locations $\phi \rightarrow-180^{\circ}$ driving the $\nabla p$ flow to the limiter which acts as a localized sink of the plasma. Consider- 
ing that the poloidal variation in the outboard region at $\phi=-180^{\circ}$ is slow, comparing the outboard density distribution at $\phi=-180^{\circ}$ with that at the limiter location, $\phi=0^{\circ}$, as plotted in Figs. 4(c) and (d) using circles, it is clear that the density values in the SOL region $\left(r>r_{0}\right)$ drop from upstream locations $\phi=-180^{\circ}$ to the downstream locations of the limiter $\phi=0^{\circ}$. This density drop could approximately be analyzed using the momentum equation, Eq. (2), of the underlying model which upon neglecting, for simplicity, the friction, viscosity etc., in a 1-dimensional setup yields [39],

$$
n_{u}\left(k T_{e u}+k T_{i u}\right)=\left(1+\frac{u_{d}^{2}}{c_{s}^{2}}\right) n_{d}\left(k T_{e d}+k T_{i d}\right),
$$

where $n_{u}$ and $n_{d}$ are the density values at upstream and downstream locations, respectively. Considering further the conditions with reasonable uniformity in the temperatures over the SOL parallel length and the flow velocity at the targets determined by the Bohm condition, $u_{d}=c_{s}$, where $c_{s}$ is the ion acoustic velocity, the reduced momentum balance (5) prescribes,

$$
n_{u}=2 n_{d}
$$

describing the drop of about a factor two in the density between the upstream (stars) and downstream (circles) locations inside the entire SOL which is the region with $r>r_{0}$.

Radial density profiles in the inboard side at these two toroidal locations are also presented in Fig. 4(c) and Fig. 4(d) using stars $(*)$. The inboard density at upstream location, $\phi=-180^{\circ}$, away from the limiter, is seen to resemble the outboard density profile. The upstream density $\left(\phi=-180^{\circ}\right)$ at both inboard and outboard sides remains higher than the density for the region $r>r_{0}$ at downstream location $\phi=0^{\circ}$ outboard side. Strikingly, the radial density at the inboard side at the limiter location $\phi=0^{\circ}$ is also seen comparable or even larger than the densities at the upstream location, $\phi=-180^{\circ}$. This is clear from the density in SOL at inboard $\phi=0^{\circ}$, plotted with stars $\left(^{*}\right)$ in Fig. 4(c), which marginally exceeds the density at the location $\phi=-180^{\circ}$ for same $r$ values, indicating that the inboard side of the limiter location could correspond to an even more upstream location such that the SOL density value tends to attain a maximum value at this location. In this respect, the connection length distribution plotted in Fig. 3(a) indeed shows the inboard poloidal location $\theta=180^{\circ}$ at the limiter plane $\phi=0^{\circ}$ to be a region of longer connection lengths as compared to the inboard region of the plane $\phi=-180^{\circ}$ plotted in Fig. 3(b). The density maximum and an apparent presence of the stagnation region to the inboard side at the limiter location, $\phi=0^{\circ}$, must result in the poloidal gradients stronger than estimated assuming $\phi=-180^{\circ}$ to be a more obvious stagnation location because of its longer physical separation from the limiter, as considered for many measurements. Since the larger density at the stagnation point relates to considerable particle source but weaker parallel transport, such a density maximum at inboard remains a strong possibility for the cases where the source in the SOL might be significant at the inboard (high field) side and comparable to that in the outboard (low field) side. Considering that in the present treatment uniform perpendicular transport coefficients, $D_{\perp}$ and $\chi_{\perp}$ are employed allowing equivalent strength of the diffusive source from the core to both the inboard as well as outboard side, however a weaker parallel transport is expected due to symmetry view point at $\phi=0^{\circ}$ inboard side, the enhanced density at this location in the simulations highlights such a possibility in the operations where similar conditions are approached.

The additional assumption of an approximate uniformity in the temperature values in the SOL for obtaining (6) can be verified by examining the corresponding electron temperature distribution computed in the SOL for the present set of operational parameters. The electron temperature distribution is also plotted at the locations $\phi=0^{\circ}$ and $-180^{\circ}$ in the Fig. 5(a) and (b), respectively, showing similarity in its values to a large extent. In order to describe the conditions more elaborately, the radial temperature profiles are presented both in the inboard and outboard regions of the two locations, $\phi=0^{\circ}$ and $-180^{\circ}$, in the Fig. 5(c) and (d), respectively. The radial profiles at all the four locations show very clear similarities indicating the absence of any parallel gradient of the electron temperature in the SOL and therefore an almost isothermal flow of plasma to the limiter target in the region for the present set of parameters, as assumed to obtain Eq. (6) from Eq. (5). Results presented in Fig. 4 and 5 highlight the basic symmetry and nonlocal structure of the plasma profiles that in turn determine the global plasma transport to zeroth order. We now turn to analyzing various local and small length scale structures in the profiles that constitute flow structure to first order in the spatial variation.

With the temperature profiles confirming the estimates for the density variation across the parallel dimensions of the SOL, we now explore the flow velocity that must vanish at the stagnation point [40]. Profiles of the parallel Mach number, $M$, derived from the simulation data are plotted in Fig. 6(a)-(d). The Mach number at the limiter location, plotted in Fig. 6(a) approaches its maximum value $M \sim 1$ beyond the LCFS $\left(r>r_{0}\right)$ while it drops to much smaller values inside the core region. Plotted at $\phi=-180^{\circ}$, away from the limiter location, $M$ however drops to much smaller value all over the region but shows an interesting pattern in its radial-poloidal distribution. A careful inspection shows signature and effects of this small but systematic variation also in the core plasma, however in the plots corresponding to the limiter location this variation is dominated by a very high offset of the unperturbed zero order flow in the SOL which is comparable to the ion sound velocity. At upstream locations like $\phi= \pm 180^{\circ}$ where the background flow drops to negligible values in comparison to $M \rightarrow 1$ at the target loca- 

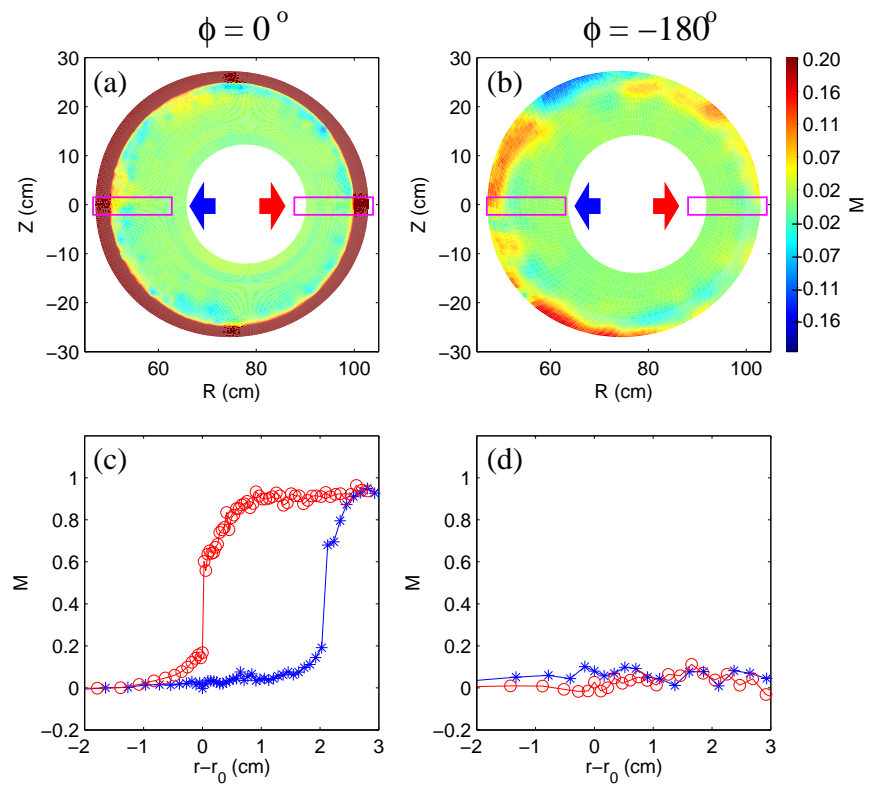

FIG. 6: 2D radial-poloidal cross-section of the simulated 3D Mach number distribution at the toroidal locations (a) $\phi=0^{\circ}$ or the limiter location and (b) $\phi=-180^{\circ}$ or the diametrically location opposite to the limiter plane. Radial profiles of the Mach number on inboard $\left(^{*}\right)$ and outboard (o) sides at toroidal locations (c) $\phi=0^{\circ}$ and (d) $\phi=-180^{\circ}$.

tion, this flow perturbation begins to be well resolved, as in Fig. 6(b). Comparing the Mach number variation with that in the density in the SOL region $\left(r>r_{0}\right)$ it is clear from Fig. 4(d) and Fig. 6(d) that the plasma away from the limiter has considerable density but negligible flow velocity. This plasma develops considerable flow velocity in its approach to the limiter as clear upon correlating the outboard profiles in Fig. 4(c) and Fig. 6(c), plotted with circles. Note that this behavior of the outboard profiles in the SOL region is in contrast to that of the inboard profiles (plotted with *) which show that while the density in the inboard remains high even at the limiter location $\phi=0^{\circ}$, the Mach number has vanishing values, indicating that the flow velocity of the plasma remains negligible at this location. The result that the SOL plasma density is marginally larger at this location in comparison to the inboard side at $\phi=-180^{\circ}$ identifies this location as further upstream to the latter.

\section{COMPARISON WITH THE PROBE MEASUREMENTS}

A quantitative agreement of the simulated profiles with probe measurements is not expected in the present level of the study where major reasons for deviation of simulated results from probe measurements exist, including (i) simplicity of the model (1)-(4), (ii) a simplistic setup where limiter is the only plasma sink, and (iii) a rather ideal, perturbation-free plasma equilibrium represented
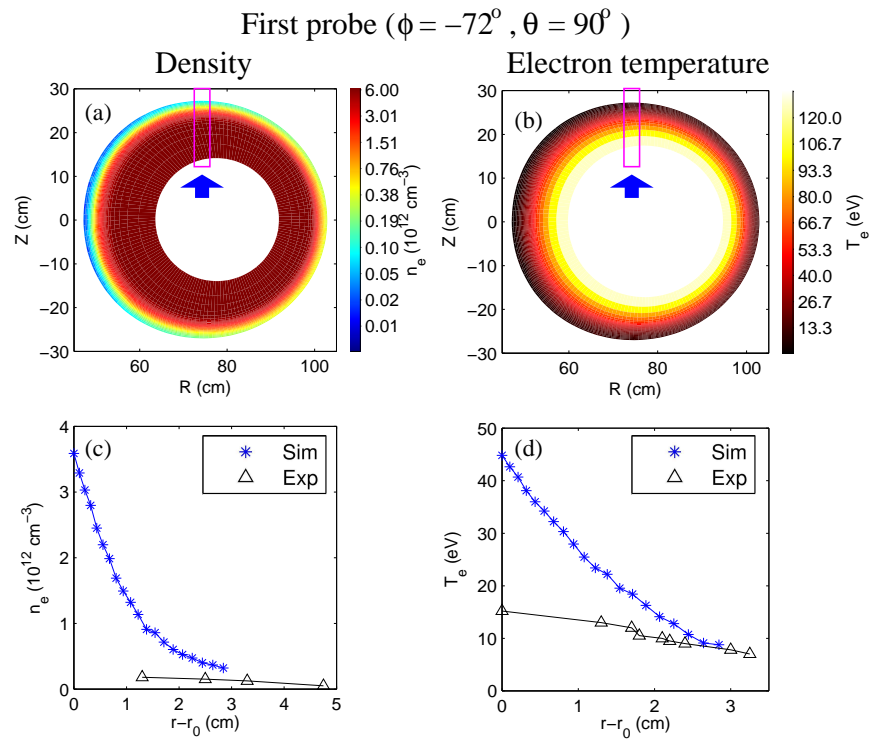

FIG. 7: 2D radial-poloidal cross-section of the simulated 3D distributions of (a) density and (b) electron temperature at the location of the top probe at $\phi=-72^{\circ}$ and $\theta=90^{\circ}$ (top of the torus). The radial profiles of the density (c) and the electron temperature (d) from the simulation $(*)$ and from the fit to data in Ref. [17] obtained from the probe measurements (triangles) at the above location.

by the 3D mesh. However, a qualitative comparison is attempted with recently reported probe measurements of the radial density and temperature variation in the SOL. As discussed in Sec. I, although qualitative in nature, the present simulation results can serve as a stronger global model in comparison with the schematic considerations that are routinely used for the interpretation of the probe measurements available only at limited locations. Simulation data for these quantities extracted from the locations corresponding to the first probe, toroidally $-72^{\circ}$ away from the limiter in the zone-I and at a poloidal angle about $90^{\circ}$ towards the top from the mid plane, is presented (using stars) along with the measured values (using triangles) in Fig. 7 (c) and (d), respectively. Fig. 7(a) and (b), show the corresponding complete 2D radial-poloidal distributions simulated of the density and temperature, respectively. A similar set for the second probe location, $\phi=54^{\circ}$ and the poloidal angle $\theta=45^{\circ}$ towards the top from the midplane, is presented in Fig. 8 (a)-(d), respectively. As seen from Fig. 8(c), the simulated density profile shows a near-agreement with the experimental measurements, however, such a quantitative agreement remains limited only to the far SOL region (large $r-r_{0}$ ). The deviation between the two in rest of the region is mainly from the reason that the SOL plasma in the simulations has an interface with a rather simplistic core plasma which the present model (optimized for $\mathrm{SOL}$ ) is capable of simulating. In comparison to this, the SOL plasma in the experiments is coupled to a more realistic core plasma having many complex attributes. 

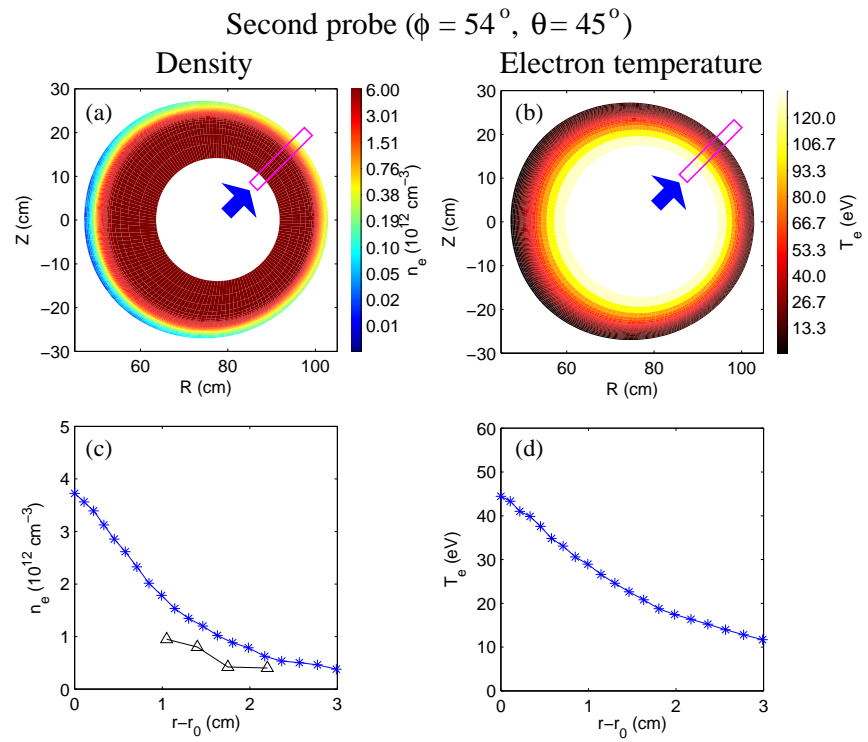

FIG. 8: 2D radial-poloidal cross-section of the simulated 3D distributions of (a) density and (b) electron temperature at the second probe location $\phi=54^{\circ}$ and $\theta=45^{\circ}$. (c) The radial profiles of the density from the simulation $\left(^{*}\right)$ and from the fit to data in Ref. [17] obtained from the probe measurements (triangles) at the above location. (d) The radial profiles of the electron temperature from the simulation $(*)$ at the above location.

The simulated Mach number values at these two probe locations are similarly compared with the radial profiles of the Mach number values measured in the experiments in Fig. 9. Both in the measurements and in the simulations the magnitude of Mach number at these two probe location has value in an intermediate range as compared to the two limiting locations, i.e., far upstream $\left(\phi=-180^{\circ}\right)$ and downstream (limiter location $\left.\phi=0^{\circ}\right)$, where the Mach number $M$ in the simulations approaches values vanishingly small and 1 (flow velocity comparable to the sound velocity), respectively, as seen in Fig. 6(d) and (c). Among the major additional first order effects expected in the rather simplistic set up of Aditya SOL plasma, that are absent from the simulation and therefore likely to be the source of deviation in the simulated Mach number profiles, are: (i) the $E_{r} \times B_{\theta}$ drift due to the radial electric field across the SOL width arising from the variations in the plasma floating potential and the electron temperature, (ii) The Pfirsch-Schluter current and flow arising from charge separation generated by the curvature effects (given that the top and bottom locations are magnetically connected through the SOL volume in Aditya unlike the SOL formed by a toroidally continuous divertor), and (iii) the Ballooning transport effect similar to that in the SOL of a single null divertor tokamak where inboard and outboard SOL regions are magnetically connected, much like in the Aditya SOL.

The simulated $M$ profiles could be compared with the usual measurements of the Mach number $[17,18]$, as in
First probe $\left(\phi=-72^{\circ}, \theta=90^{\circ}\right)$ Second probe $\left(\phi=54^{\circ}, \theta=45^{\circ}\right)$
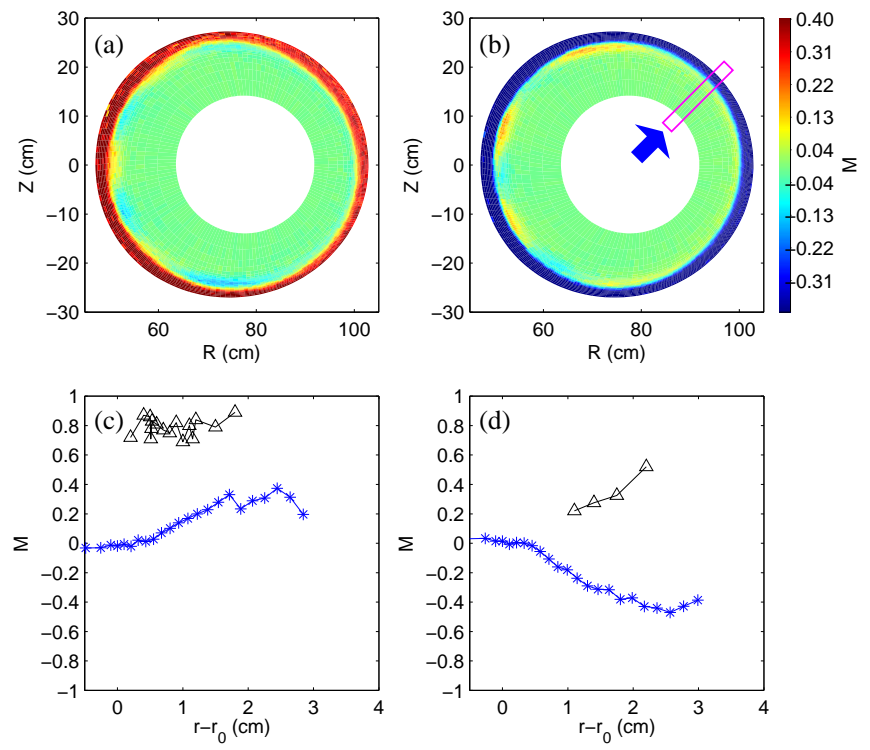

FIG. 9: 2D radial-poloidal cross-section of the simulated 3D distributions of the toroidal Mach number at the location of (a) the first probe at $\phi=-72^{\circ}$ and $\theta=90^{\circ}$ (top probe). and (b) the second probe at $\phi=54^{\circ}$ and $\theta=45^{\circ}$ (radial probe). (c) The radial profiles of $M$ from the simulation (*) and from the fit to data in Ref. [17] obtained from the probe measurements (triangles) at the first probe location. (d) The radial profiles of $M$ from the simulation $\left(^{*}\right)$ and from the fit to data in Ref. [17] obtained from the probe measurements (triangles) at the second probe location.

Fig. 9, for the configuration where both, the magnetic field $B$ and the plasma current $I_{p}$, are clockwise (CW, CW) when seen from the top [22]. The points (triangle) in Fig. 9 (c) and (d) correspond to the routine observation in Aditya SOL that - on the first probe the $M$ value is large positive ( $\equiv$ clockwise when seen from the top) while on the second probe flow is small positive. This is consistent with a clockwise drift contribution in the Mach number, $+M_{d}$, at both probe locations and the contributions of the transport flow values $\pm M_{T}$ at the first and second probe locations, respectively, with $\left|M_{d}\right|>\left|M_{T}\right|$ (here the negative sign corresponds to counter-clockwise direction) such that the Mach number values at the first probe are,

$$
M_{I}^{\mathrm{CW}}=M_{d}+M_{T} \quad \text { (large positive), }
$$

while at the second probe,

$$
M_{I I}^{\mathrm{CW}}=M_{d}-M_{T} \quad \text { (small positive), }
$$

as correctly expected in this set up, where superscript ' $\mathrm{CW}$ ' indicates the measurements in the configuration (CW, CW). A more recent set of experimental observations, using the technique of reversal of the orientations of both the magnetic field $B$ and the plasma current $I_{p}$ to counter clockwise direction $(\mathrm{CCW}, \mathrm{CCW})$, 
Ref. [22, 41, 42], has indicated that the drift effects might indeed constitute a dominant part of the net plasma flow in the device SOL. This reversal of both $B$ and $I_{p}$ is additionally reported to cause the net toroidal flow measured at the first and second probe locations to be 'small positive' and 'small negative', respectively, which is in deviation from the expected result (considering that up on such a reversal the drift contribution becomes counterclockwise, $M_{d} \rightarrow-M_{d}$, while $M_{T}$ is unaffected) that at the first probe location,

$$
M_{I}^{\mathrm{CCW}}=-M_{d}+M_{T} \quad \text { (small negative) }
$$

while at the second probe,

$$
M_{I I}^{\mathrm{CCW}}=-M_{d}-M_{T} \quad \text { (large negative) }
$$

This deviation from (9) and (10) in the probe data shows that, if the drift magnitude is indeed unchanged, the transport components measured by two probes may have an imbalance in the magnitude, in at least one of the two experimental set ups with different orientations. This imbalance can be attributed either to a net rotation of the plasma [43] or the fact that the probes are at different poloidal locations and sample the strong poloidal nonuniformity of the transport driven flows as computed in the present EMC3-EIRENE simulations as discussed further in the Sec. VI.

In the light of above discussion, the simulation results and their comparison with the measurements presented above provide quantitative inputs and feedback for systematically including various additional effects of the drifts and flows in the model (1)-(4) in the advanced phases of the Aditya SOL transport modeling using EMC3-EIRENE. In general, considering that the process of refinement of the SOL simulation model requires a thorough benchmark with the experimental measurements carried out in rather simpler geometries, the Aditya plasma presents an ideal setup with its simplistic circular plasma cross-section and an associated SOL with equally simpler and easy to interpret drift structure. The presented comparison from the first phase of EMC3EIRENE simulation for Aditya and advanced phases of this activity is also expected to generate important inputs for the process of SOL modeling.

\section{FLOW MODULATIONS IN THE SOL}

The 2D distribution of the parallel Mach number $M$ at the far upstream and probe locations presented in Sec. IV and $\mathrm{V}$ show a predominant modulation in the value along the poloidal $(\hat{\theta})$ direction in the region of SOL. This is evident in the form of a systematic pattern in the $2 \mathrm{D}$ surface plots of $M$ that are nearly anti-symmetric about the inboard midplane, or the poloidal angle $\theta=0^{\circ}$. From the associated radial profiles presented in Fig. 9 and 6 approximate magnitude of this modulation can be esti-
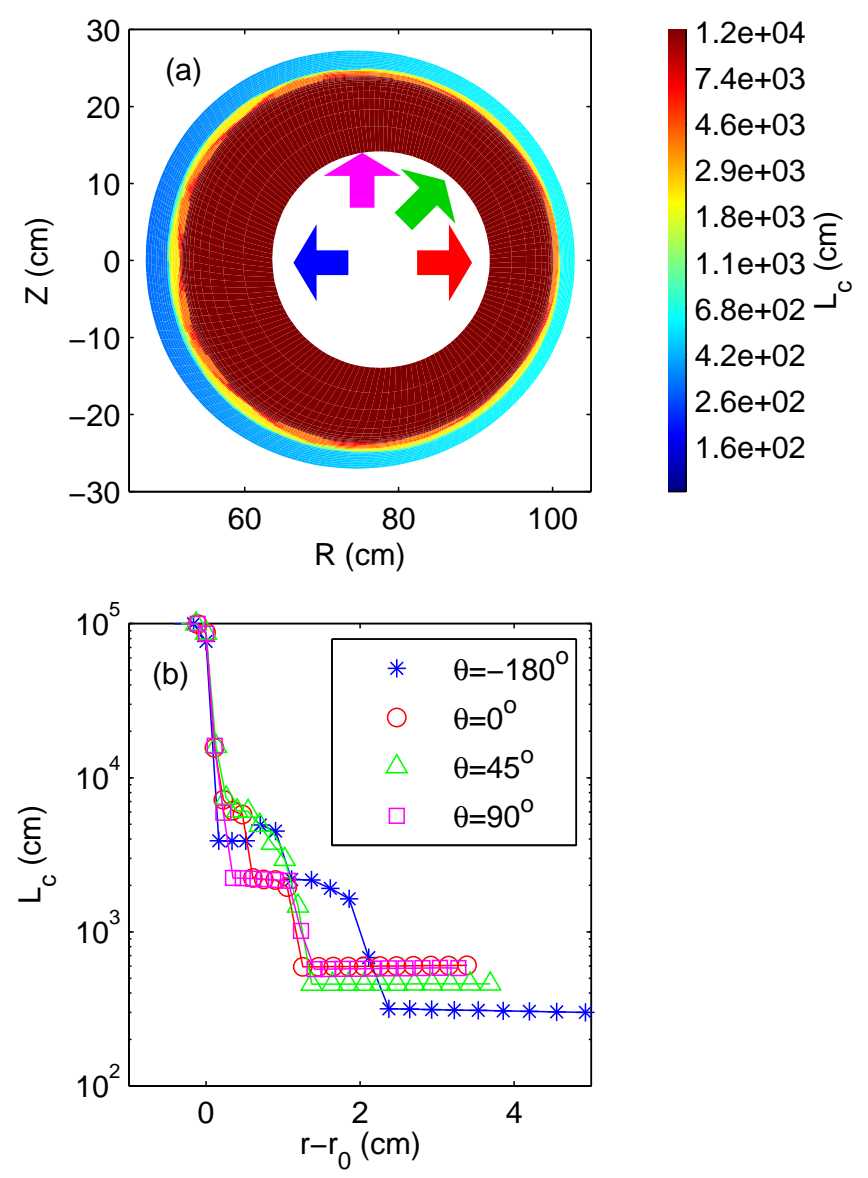

FIG. 10: (a) Distribution of the parallel connection lengths, $L_{c}(r, \theta)$, on the radial-poloidal plane at the far upstream toroidal location $\phi=-180^{\circ}$. (b) Radial profile of the connection length in the SOL for above toroidal location, at poloidal locations $\theta=-180^{\circ}$ (inboard), $\theta=0^{\circ}$ (outboard), $\theta=45^{\circ}$ (radial probe angle), $\theta=90^{\circ}$ (top probe angle), respectively.

mated to be about 0.1 to 0.2 , i.e., the flow strength of about 10 to $20 \%$ of the ion sound velocity. Since the $M$ changes sign along $\theta$ the relative flow velocity between the two parallel streams of plasmas can approach up to $40 \%$ of the ion sound speed. Further, at the toroidal locations close to the limiter (e.g., in Fig. 6(a)) the modulation is fairly dominated by a large offset in $M$ value present beyond the limiter radius due to a strong radial jump in the $M$ value to 1 from a vanishing bulk value while at the smaller radius remains visible due to absence of such an offset. Similarly, at the toroidal locations away from the limiter (e.g., in Fig. 6(b)), where no region of large $M$ value offset is present, the modulations are visible throughout the SOL region. Let us now consider the fact that $M$ value is the only function which is free from a large offset in certain regions of the plasma and therefore allows the modulations to be visible in the distributions plotted in such regions. The presence of structures in the flow profiles can readily be attributed to a complex dis- 
tribution of connection length values in the SOL region as plotted on one of the $2 \mathrm{D}$ cross-sections $\left(\phi=-180^{\circ}\right)$ of the torus presented in Fig. 10(a) where the presence of a wider transition region for connection length from a minimum value $\left(L_{c} \sim 2 \pi R \pm r_{0}\right)$ to very high value is evident having a finite poloidal variation. This transition region ranges from the LCFS to the far SOL region. As seen from the radial profiles of the connection length plotted in Fig. 10(b) the radial jump in the connection length value is sharpest in the radial profile corresponding to the outboard poloidal location $\theta=0^{\circ}$, while at other poloidal locations it occurs over a much wider range of radii, covering almost the entire width of the SOL, for example, in the profiles corresponding to the inboard side $\left(\theta=-180^{\circ}\right)$ and at the probe locations $\left(\theta=45^{\circ}\right.$ and $\left.90^{\circ}\right)$. Moreover, the connection length value is seen to have a radial fluctuation for the profile corresponding to $\theta=-180^{\circ}$ plotted with ' $*$ ' in Fig. 10(b).

The equilibrium forms of continuity of particle and momentum suggest that associated with the modulations recovered in the equilibrium Mach number values $M$, the modulation must be present in the plasma density. Such density modulations however remain rather invisible, both from the radial profiles and the $2 \mathrm{D}$ distributions of the plasma density, mainly due to the reason that a large density offset is present everywhere and, unlike the Mach number variation, there is no region in the SOL where the unperturbed density value vanishes and the small amplitude density modulations begin to become visible.

The poloidal variation along the LCFS in the $M$ values at the toroidal locations $\phi=-180^{\circ}$ (far upstream) $\phi=-72^{\circ}$ (top probe) $\phi=0^{\circ}$ (limiter) and $\phi=54^{\circ}$ (radial probe) are presented in the Fig. 11 where a quasiperiodic variation is seen to exist in the Mach number values which is nearly antisymmetric about the inboard midplane, except a poloidal shift in the point of symmetry due to the finite $q$-value at the LCFS. The $M$ values at the probe locations, $(\phi, \theta)=\left(-72^{\circ}, 90^{\circ}\right)$ and $\left(54^{\circ}, 45^{\circ}\right)$ are such that they must have positive and negative contributions in the Mach number measurements by the top and radial probes, respectively, (effectively, the $M_{T}$ values in Eq. (7)-(10)). This to a large extent explains the difference in the positive values in the Mach number measured in the experiments by both these probes, since these contributions from pressure driven transport must effectively be added to the large positive contribution coming from the $E_{r} \times B_{\theta}$ drift and the PfirschSchluter (PS) flow values which are the additional effects absent from the simulation model. The parallel Mach number $M$ value for the limiter location, $\phi=0^{\circ}$, has a modulation comparable to other toroidal angles everywhere except in a small poloidal range about $\theta=0^{\circ}$ which corresponds to the position of the plasma strike point on the limiter and where the $M$ jumps to a considerably high value $(\sim 0.7-0.8)$. Although LCFS is a surface with closed field lines, the plasma in this poloidal range $\left(-16^{\circ}<\theta<22^{\circ}\right)$ acquires a large parallel velocity
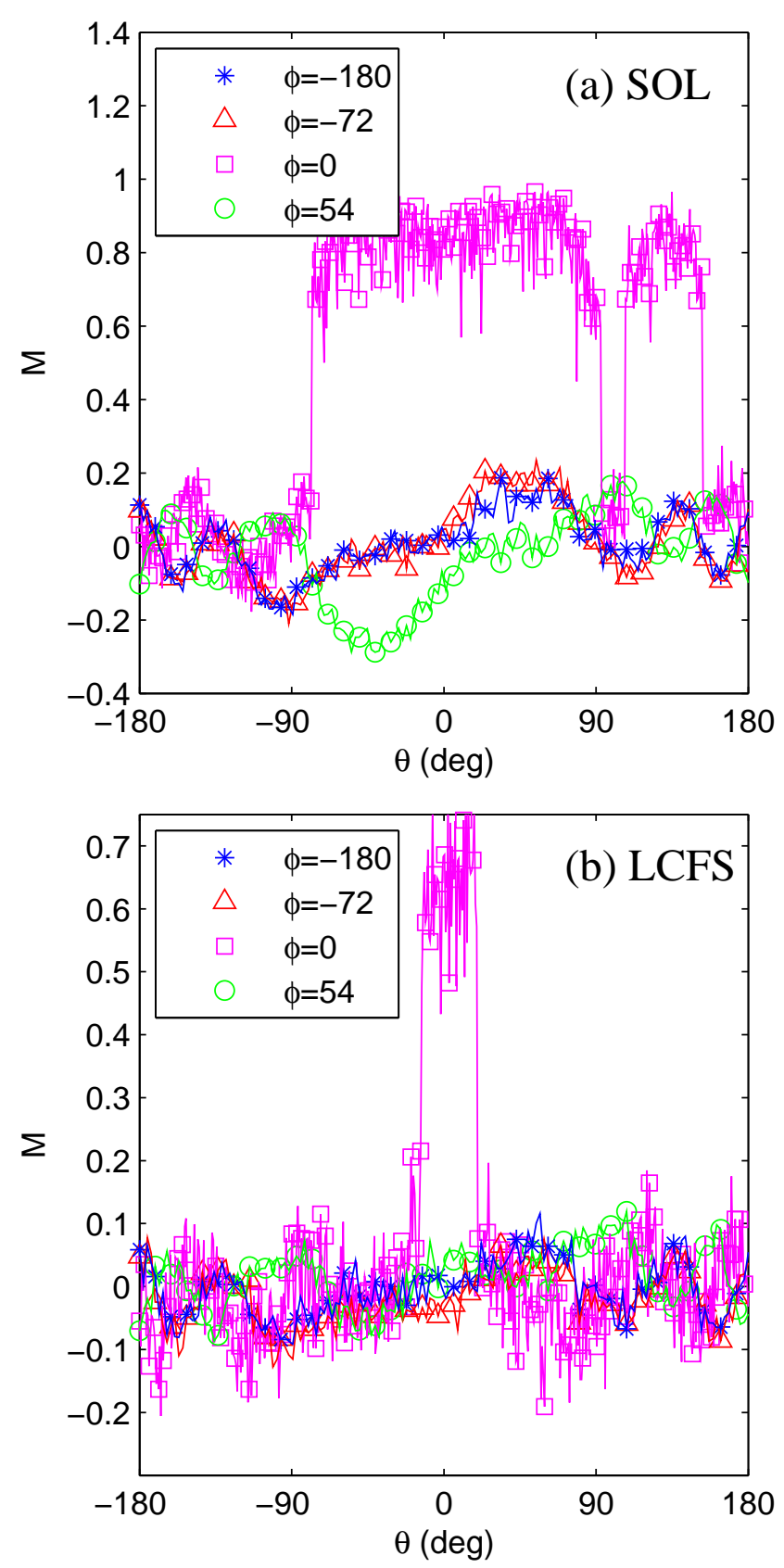

FIG. 11: Simulated poloidal variation of Mach number $M$ (a) in the SOL and (b) on the LCFS, at the toroidal locations corresponding to the far upstream $\left(\phi=-180^{\circ}\right)$, top probe $\left(\phi=-72^{\circ}\right)$, limiter $\left(\phi=0^{\circ}\right)$ and radial probe $\left(\phi=54^{\circ}\right)$.

via a perpendicular diffusion of the parallel momentum (finite perpendicular viscosity $\eta_{\perp} \sim m_{i} n D$ in Eq. 2) from the plasma on the next magnetic surface that belongs to SOL and has a flow approaching $M=1$ due to presence of limiter with narrow striking zone having almost same poloidal extension. It can be noted that this poloidal perturbation is very sharp at the toroidal location of the limiter and localized in the region of the plasma strike-point, it is however more diffused at relatively downstream lo- 

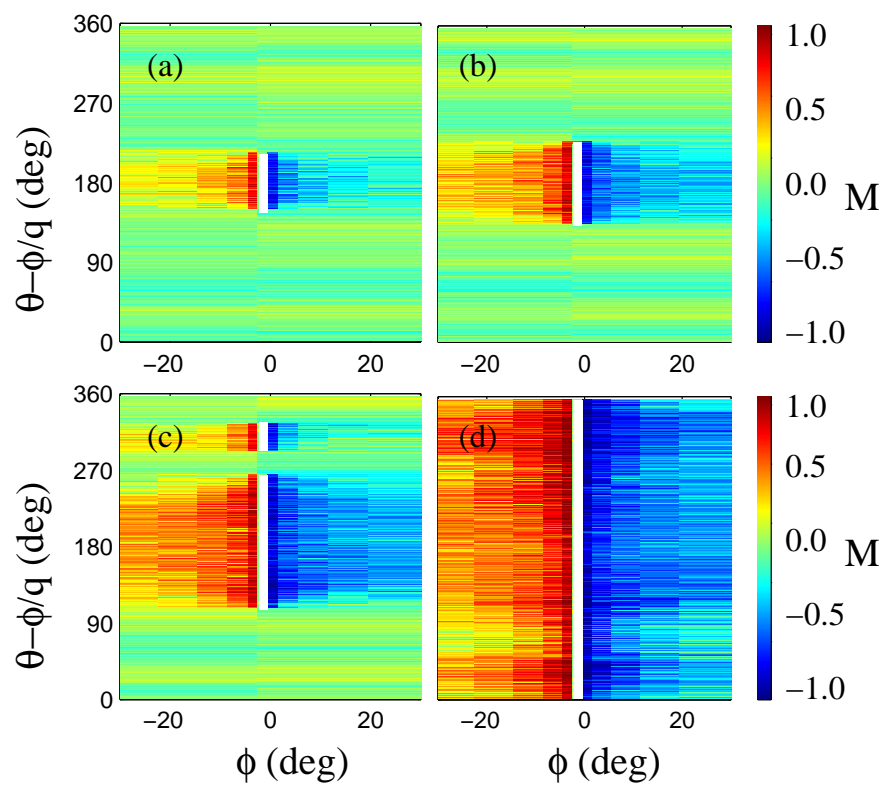

FIG. 12: The distribution of plasma Mach number on toroidal-poloidal magnetic surfaces with increasing radius in zone-II. Plots (a) - (d) correspond to radial index, $n_{r}=28$, 35,45 and 52 respectively, the circular outer radial boundary of the simulation zone corresponds to $n_{r}=72$ for zone-II. The lighter patches at $\phi=0^{\circ}$ represent the part of limiter ring intersected by the surface.

cations once again due to effect of a finite perpendicular viscosity $\eta_{\perp}$. The variation in the simulated Mach number at both the probe locations also corresponds to the magnitudes of the $M$ measured in the experiments which recovered a variation indicating the transport component relatively smaller at the top probe as compared to the radial probe. A secondary peak in the Mach number distribution in the SOL profiles (plotted in Fig. 11(a)) is attributed to increasing magnitude of the marginally asymmetric ripples in the magnetic flux surfaces of higher radial index (originating from the square configuration of the toroidal field coils about the vessel) which are likely to intersect the limiter at multiple poloidal locations (see Fig. 3). However the plasma density on these radially outer surfaces is considerably smaller as compared to the LCFS values in the profiles plotted in Fig. 11(b), the plasma Mach number there must approach its boundary value, $M=1$, causing the additional peak.

In order to illustrate the toroidal form of the structures whose poloidal variation is visible in the Mach number distributions presented in Fig. 6 and 9, we have presented in Fig. 12 the distribution of $M$ on various toroidal-poloidal planes located in the zone-II that are separated radially and include the magnetic flux surface representing the LCFS in the simulation (i.e., the surface containing cells which have first intersection with the limiter). In Fig. 13, we have plotted the poloidal profiles of the density along the LCFS using the same set of selected toroidal locations as used for Mach number $M$
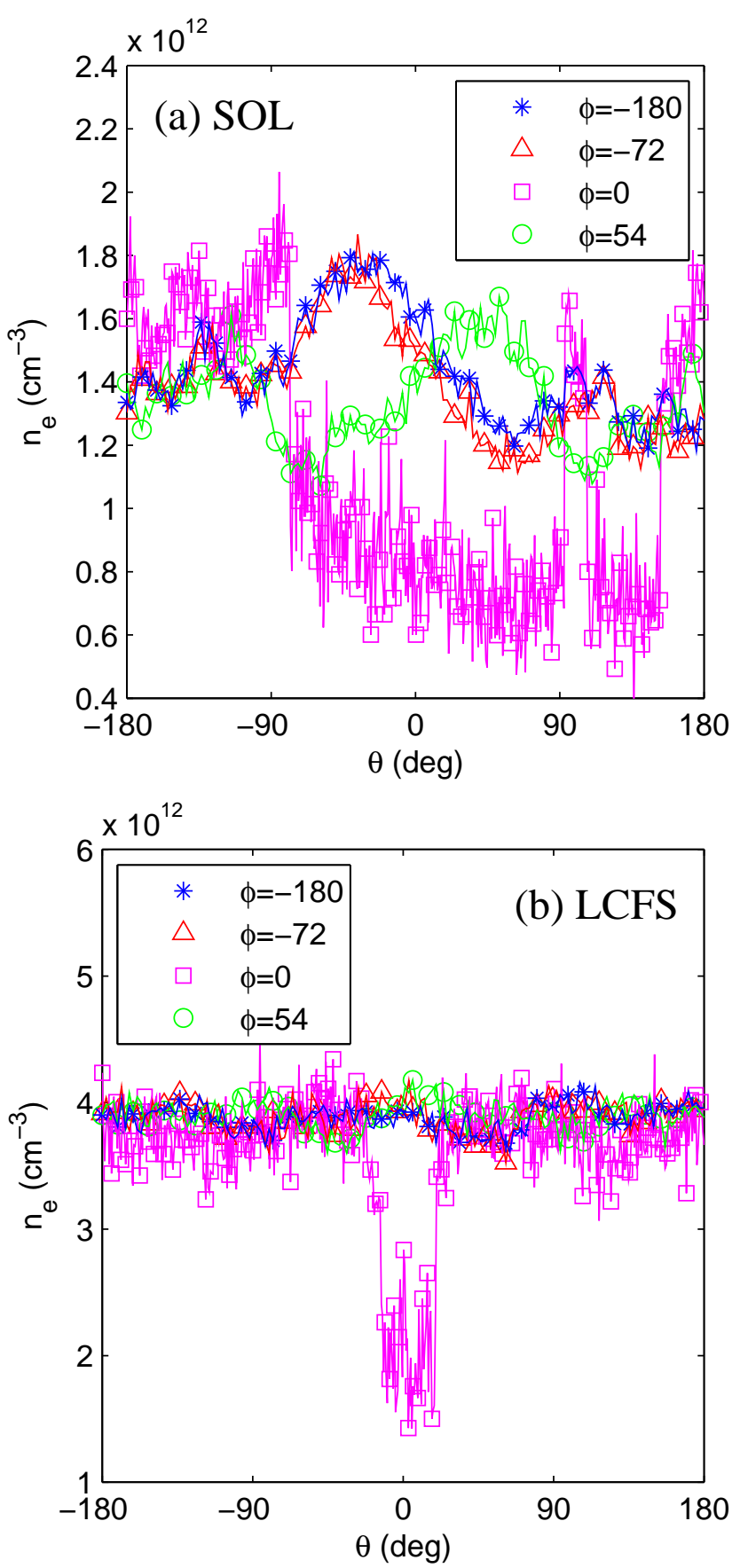

FIG. 13: Simulated poloidal variation of density $n_{e}$ (a) in the SOL and (b) on the LCFS, at the toroidal locations corresponding to the far upstream $\left(\phi=-180^{\circ}\right)$, top probe $\left(\phi=-72^{\circ}\right)$, limiter $\left(\phi=0^{\circ}\right)$ and radial probe $\left(\phi=54^{\circ}\right)$.

in Fig. 11, i.e., $\phi=-180^{\circ}$ (far upstream) $\phi=-72^{\circ}$ (top probe) $\phi=0^{\circ}$ (limiter) and $\phi=54^{\circ}$ (radial probe). At the limiter location, $\phi=0^{\circ}$, the poloidal variation of the density is very sharp with a net drop $\sim 2 \times 10^{12} \mathrm{~cm}^{-3}$ which confirms with the drop of density from upstream to downstream locations by a factor of 2 as prescribed by the Eqs. (5) and (6) obtainable from the simulation 
model. The density profiles at both the probe locations and the location $\phi=-180^{\circ}$ show density values much larger compared to the limiter location and also show a very systematic poloidal variation of quasiperiodic nature.

We observe that for the simulations performed keeping in view a selected discharge scenario, while parameters like edge density or recycling flux can be varied with a limited freedom, the information of the magnetohydrodynamic equilibrium is frozen in the $3 \mathrm{D}$ mesh used to perform the transport computation. Since the toroidal magnetic field and plasma currents determine the MHD equilibrium and, in turn, the 3D mesh or $q$ values for the computations, the observed flow structures whose topology is largely determined by the plasma $q$ can be considered unaffected over a considerable range of plasma parameters like density and additional flows that are routinely recovered in the experiments. The flow structures present an example of the possible impact of the modified slab model, which must replace its former schematic version, and is required in order to interpret the present simulation results that accounts for the relative curvature of the equilibrium magnetic surfaces and the poloidally continuous limiter. The flow structures must form as the finite relative curvature between LCFS and limiter leads to surfaces that are partly open since they have a small poloidal intersection with the poloidal limiter of a different curvature. An additional condition for the existence of structures is an appropriate ratio of the strength of the parallel and perpendicular transport processes in the SOL region such that the limiter shadow is a toroidally extended sink of the plasma. In this view the impact of perpendicular transport coefficients on the structure topology needs to be analyzed in further detail in future studies.

An already expected consequence of the observed filamentation of the parallel flows in LCFS region and the associated poloidal modulation in the density is their direct impact on the continuity of the flow shear in the edge region. The simulated flow profiles and their impact, via an associated density modulation capable of generating secondary drifts, are likely to contribute to the divergence of the routinely expected drift and flows, including those measured in Aditya SOL. Although the effect of these drifts are absent from the present simulation model, the perturbations in their intensity arising from the secondary drifts generated by the density modulations can be readily estimated from the present simulation data. For example, given that both the temperature and density profiles are available from the simulations to lowest order, the intensity of the radial component of the diamagnetic current density arising the poloidal perturbations present in resulting pressure profile can be given on the LCFS as

$$
\mathbf{j}_{\mathbf{d}}=\frac{\mathbf{B} \times \nabla n\left(T_{e}+T_{i}\right)}{B^{2}} .
$$

Considering that both the radial and poloidal compo-
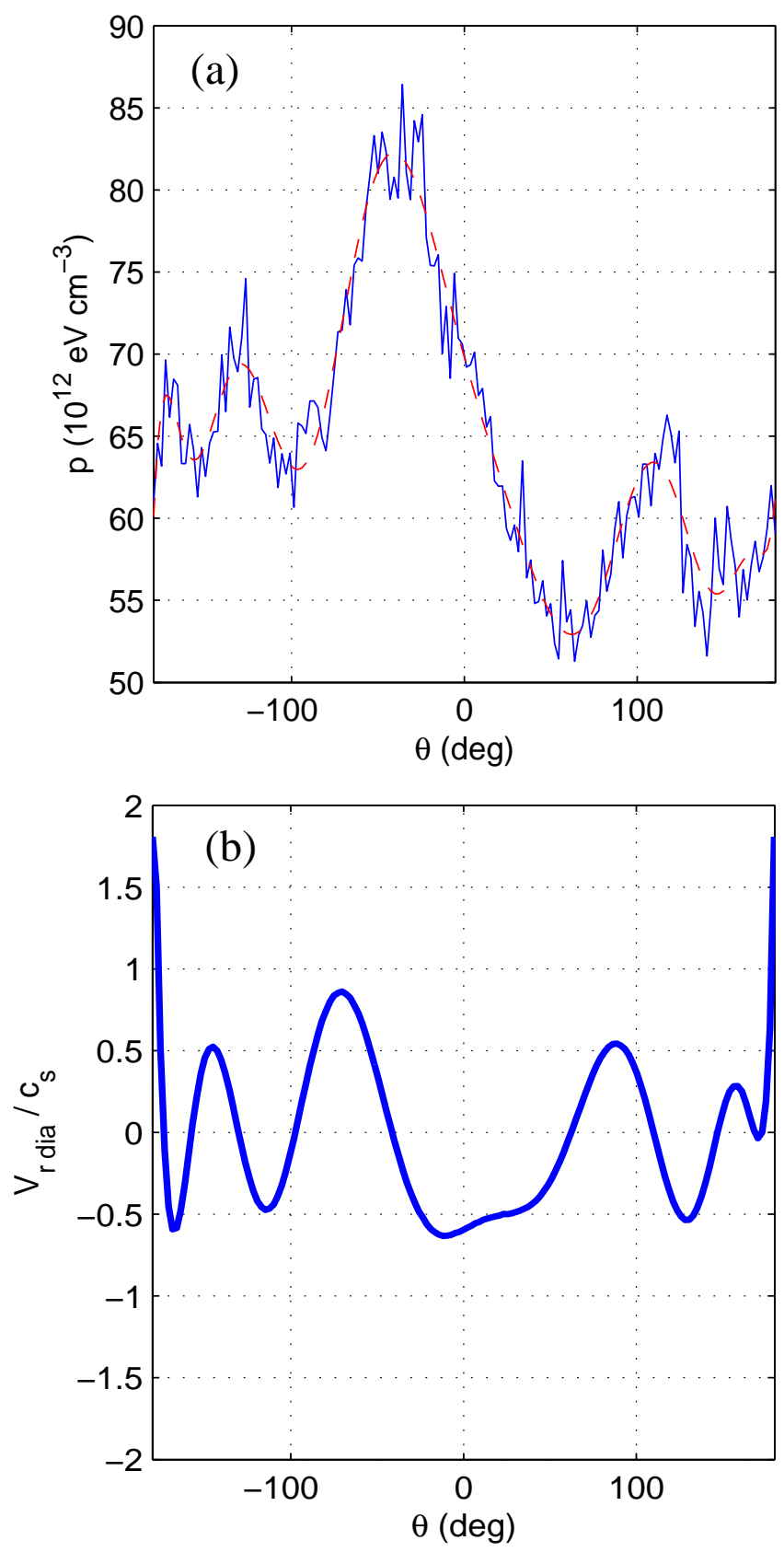

FIG. 14: Simulated poloidal variation of (a) the pressure $p$ (solid blue line) with analytic fit (dashed red line) and (b) the radial diamagnetic flow $v_{d i a}$ normalized to the ion sound speed $c_{s}$, in the SOL region at the first probe location (top probe) $\phi=-72^{\circ}, \theta=90^{\circ}$.

nents of the plasma pressure is finite in the separatrix region, possibility of both the poloidal and toroidal flow generation may be discussed due to static flows as well as the associated Reynolds stress effect [44-46] that requires finite correlations $\left\langle\delta M_{i} \delta M_{j}\right\rangle$, where index $i \neq j$ represent directions. The poloidal component of the pressure gradient, $\nabla_{\theta} p$, associated with the poloidal component of the density gradient, $\nabla n$, (plotted in Fig. 13) is presented in 


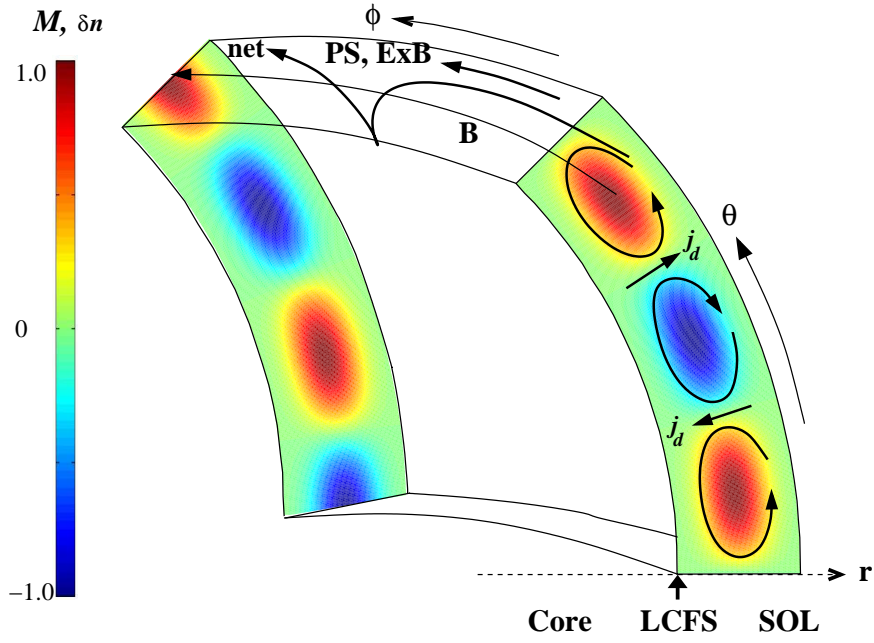

FIG. 15: Schematic view of the vorticity generation by diamagnetic flow arising from the SOL flow structures.

Fig.14(a). This poloidal pressure perturbation in combination with the toroidal magnetic field $\mathbf{B}$ is estimated to produce a finite radial component of the diamagnetic current density, $j_{\mathrm{d} r}$, given by (11), which is expected to results in the formation of zones of self-terminating diamagnetic flow in the SOL [24] whose dimensions must scale with the spatial scale of the parallel flow structures. The variation in the SOL of the intensity of the resulting radial diamagnetic drift, $v_{\text {dia }}$, normalized to the ion sound speed $c_{s}$ is plotted in Fig. 14(b) as computed using (11) and the analytic fit to simulated density data (Fig. 14(a)) at the location of the first probe. Note that this normalized radial diamagnetic flow velocity may attain a considerable value, comparable to the parallel Mach number $M$, at the poloidal locations of the maximum shear (e.g., $\theta=180^{\circ}$ ) in the parallel flow generated due to the SOL flow structures.

Since the radial diamagnetic drift splits the flow in poloidal zones, it enters the flow continuity with the primary parallel flows in the SOL, namely the $E_{r} \times B_{\theta}$ and PS flows, to generate a finite vorticity in the net SOL flows which are originally along the magnetic flux surface but must cause a net cross field transport in presence of flow structures generating the diamagnetic flow. This mechanism is illustrated in further detail in the schematic diagram presented in Fig. 15 where the poloidally alternating diamagnetic flows are shown to be responsible for generation of a density perturbation $\delta n$, which, in turn, produces an extra cross-field circulation in the above parallel SOL flows via generating $\mathbf{j}_{\mathbf{d}}$ given by Eq. 11 . This process must affect the degree of turbulence in the SOL influencing the cross field diffusivity $D_{\perp}$ in the region. Since this mechanism is localized in the SOL and the generated $j_{d}$ decays in approach to LCFS, a finite effect of this process can therefore be expected on the ratio of perpendicular (anomalous) diffusivities, $D_{\perp \text { edge }} / D_{\perp \text { SOL }}$, in the edge and SOL regions which is among key parame- ters determining the pedestal properties. The strength of the structures therefore directly relates to strength of the radial electric field or the edge transport barrier since a jump in the $D_{\perp}$ across the LCFS is strongly correlated to the pedestal height. We finally discuss that though the present simulations prescribe the equilibrium parameter profiles, the stability of these equilibria is subject to detailed stability analysis. While the considerable parallel and perpendicular flow shear recovered in these equilibrium profiles is likely to make them unstable with respect to various shear driven instabilities, the quasi periodic poloidal perturbation of the flow can be a potential trigger for the equilibrium modes and similarly for the instabilities of a strong interface at the LCFS that result into processes involving large scale radial flows, like blob formation.

\section{SUMMARY AND CONCLUSIONS}

We presented detailed analysis of the results of 3D plasma transport simulation in the SOL generated by a poloidally continuous and toroidally localized ring-like limiter in the tokamak Aditya having a circular plasma equilibrium. The analytic model underlying EMC3EIRENE simulates flows driven by the diffusive source from the core and resulting pressure gradients driving the net plasma flow to the narrow strike-zone at the lowfield-side of the limiter that acts as a localized sink of the plasma. These results allow exploration of strength and structure of the drifts and curvature driven flows up on comparison with the probe measurements on the device since signatures of these features are additionally present in the probe measurements. The $3 \mathrm{D}$ computation results having a global character complement the limited but sufficiently robust probe measurements available from a set of 3 probes mounted at the known locations on the device SOL. The EMC3-EIRENE transport computations being based on 3D mesh drawn from an MHD equilibrium data allow systematic improvement in existing interpretation of the probe data where a rather schematic slab model had been in use for representing the 3D SOL region $[17,18,22]$.

The major conclusions from the present analysis include that (i) there is a significant drift and curvature driven flow contribution in the probe measurements. (ii) The recent experimental efforts to extract the transport driven flow from the net flow measurements (by affecting a helicity reversal in the magnetic configuration) can be refined to a great extent by identifying and systematically compensating the additional strong poloidal variation present in the probe measurements drawn from the probes since they are located at significantly different poloidal locations from each other. It is apart from a desired toroidal separation introduced between these probes in order to produce a self-compensating helicity dependence in the drift components measure by them in order to obtain the transport driven flow using their av- 
erage measurements. (iii) The radial-poloidal filamented structure of the transport driven flow shows an associated poloidal variation in the plasma parameters allowing one to estimate the pressure variation and the resulting selfterminating diamagnetic flows in the SOL. Similarly, the associated diamagnetic drift estimates indicate possible presence of significant level of modulations in the known SOL flows in the device SOL affecting the nature of radial transport in the SOL region with respect to that in the adjoining edge region. These modulations influence, in turn, the relative degrees of anomalous transport across the LCFS region or the pedestal location. (iv) The analysis also indicates the possibility of observed flow modu- lations being strong enough to trigger the instabilities of the equilibrium and affect the overall confinement properties in the edge and pedestal region.

Finally, the present comparison of the results of 3D EMC3-EIRENE simulations for the simplistic circular plasma configuration of Aditya obtained using an analytic model, that excludes the drift effects, with the robust probe measurements made on the device, must produce important inputs in order to benchmark and finetune the analytic modification required in the computational model account for the drift effects with sufficient accuracy.
[1] R. Schneider, X. Bonnin, K. Borrass, D. P. Coster, H. Kastelewicz, D. Reiter, V. A. Rozhansky, and B. J. Braam, Contributions to Plasma Physics 46, 3 (2006).

[2] D. Coster, X. Bonnin, G. Corrogan, G. S. Kirnev, G. Mathews, and J. Spence, J. Nucl. Mater. 337-339, 366 (2005).

[3] Y. Feng, F. Sardei, and J. Kisslinger, J. Nucl. Mater 266-269, 812 (1999).

[4] D. Reiter, Tech. Rep. Technical Report Juelich-1947, KFA, Juelich, Germany (1984).

[5] P. J. Harbour and G. Proudfoot, J. Nucl. Mater. 121, 222 (1984).

[6] G. Proudfoot, P. J. Harbour, J. Allen, and A. Lewis, J. Nucl. Mater. 128-129, 180 (1984).

[7] J. Allen and P. J. Harbour, J. Nucl. Mater. 145-147, 264 (1987).

[8] N. Smick, B. Labombard, and C. S. Pitcher, J. Nucl. Mater. 337-339, 281 (2005).

[9] L. G. Askinazi, V. A. Kornev, S. V. Krikunov, S. V. Lebedev, A. I. Smirnov, A. S. Tukachinsky, M. I. Vildjunas, and N. Zhubr, Nucl. Instrum. Methods Phys. Res. A 623, 664 (2010).

[10] B. LaBombard, J. E. Rice, A. E. Hubbard, J. W. Hughes, M. Greenwald, J. Irby, Y. Lin, B. Lipschultz, E. S. Marmar, C. S. Pitcher, et al., Nuclear Fusion 44, 1047 (2004).

[11] H. W. Muller, V. Bobkov, A. Herrmann, M. Maraschek, J. Neuhauser, V. Rohde, A. Schmid, M. Tsalas, and Asdex Upgrade Team, J. Nucl. Mater. 363-365, 605 (2007).

[12] P. B. Snyder, R. J. Groebner, J. W. Hughes, T. H. Osborne, M. Beurskens, A. W. Leonard, H. R. Wilson, and X. Q. Xu, Nucl. Fusion 51, 103016 (2011).

[13] C. S. MacLatchy, C. Boucher, D. A. Poirier, J. Gunn, B. L. Stanfield, and W. W. Zuzak, J. Nucl. Mater. 196198, 248 (1992).

[14] B. LaBombard, J. E. Rice, A. E. Hubbard, J. W. Hughes, M. Greenwald, R. S. Granetz, J. H. Irby, Y. Lin, B. Lipschultz, E. S. Marmar, et al., Phys. Plasmas 12, 056111 (2005).

[15] F. Wagner, Phys. Rev. Lett. 53, 1453 (1984).

[16] J. Hugill, Plasma Phys. Control. Fusion 42, R75 (2000).

[17] D. Sangwan, R. Jha, J. Brotankova, and M. V. Gopalkrishna, Phys. Plasmas 19, 092507 (2012).

[18] D. Sangwan, R. Jha, J. Brotankova, and M. V. Gopalkrishna, Phys. Plasmas 20, 062503 (2013).

[19] D. Sharma, R. Jha, Y. Feng, and F. Sardei, J. Nucl.
Mater. S554-S558, 438 (2013).

[20] R. Jha, P. K. Kaw, S. K. Mattoo, C. V. S. Rao, Y. C. Saxena, and Aditya Team, Phys. Rev. Lett. 69, 1375 (1992).

[21] R. Jha, A. Sen, P. K. Kaw, P. K. Atrey, S. B. Bhatt, K. Tahiliani, R. L. Tanna, and Aditya Team, Plasma Phys. Control. Fusion 51, 095010 (2009).

[22] D. Sangwan, R. Jha, J. Brotankova, and M. V. Gopalkrishna, Phys. Plasmas 21, 062512 (2014).

[23] N. Smick, B. LaBombard, and I. H. Hutchinson, Nuclear Fusion 53, 023001 (2013).

[24] A. V. Chankin and P. C. Stangeby, Plasma Phys. Control. Fusion 36, 1485 (1994).

[25] R. Srinivasan and S. P. Deshpande, Tech. Rep. Technical Report-2007, IPR/RR-393/2007 (AUGUST, 2007), IPR, Gandhinagar, India (2007).

[26] J. L. Johnson, H. E. Dallied, and J. M. Greene, Journal of Computational Physics 32, 212 (1979).

[27] M. Kobayashi, Y. Feng, A. Loarte, G. Federici, G. Strohmayer, M. Shimada, F. Sardei, D. Reiter, and M. Sugihara, Nucl. Fusion 47, 61 (2007).

[28] R. A. Pitts, J. Horacek, W. Fundamenski, O. E. Garcia, A. H. Nielsen, M. Wischmeier, V. Naulin, and J. J. Rasmussen, J. Nucl. Mater. 363-365, 505 (2007).

[29] Y. Feng, F. Sardei, and J. Kisslinger, Phys. Plasmas 12, 052505 (2005).

[30] T. Lunt, Y. Feng, M. Bernert, A. Herrmann, P. de Marne, R. McDermott, H. W. Muller, S. Potzel, T. Putterich, S. Rathgeber, et al., Nucl. Fusion 52, 054013 (2012).

[31] Y. Feng, F. Sardei, and J. Kisslinger, Nucl. Fusion 46, 807 (2006).

[32] D. Sharma, Y. Feng, F. Sardei, and D. Reiter, Nucl. Fusion 45, 825 (2005).

[33] M. Kobayashi, Y. Feng, F. Sardei, and D. Reiter, J. Nucl. Mat. 363-365, 294 (2007).

[34] D. Hartinag, D. Reiter, Y. Feng, O. Schmitz, D. Reiser, and H. Frerichs, Contrib. Plasma Phys. 48, 1 (2008).

[35] H. Frerichs, D. Reiter, O. Schmitz, T. E. Evans, and Y. Feng, Nucl. Fusion 50, 034004 (2010).

[36] O. Schmitz, M. W. Jakubowski, H. Frerichs, D. Harting, M. Lehnen, B. Unterberg, S. S. Abduallaev, S. Brezinsek, I. Classen, T. Evans, et al., Nucl. Fusion 48, 024009 (2008).

[37] D. Harting, S. Wiesen, H. Frerichs, D. Reiter, P. Borner, Y. Feng, and JET-EFDA. contributors, J. Nucl. Mat. 
415, S540 (2011).

[38] J. D. Lore, J. M. Canik, Y. Feng, J. W. Ahn, R. Maingi, and V. Soukhanovskii, Nucl. Fusion 52, 054012 (2012).

[39] P. C. Stangeby, The Plasma Boundary of Magnetic Fusion Devices (Ch. 10, pp. 419, Ch. 11, pp. 430) (Institute of Physics Pub., 2000).

[40] J. P. Gunn, C. Boucher, M. Dionne, I. Duran, V. Fuchs, T. Loarer, I. Nanobashvili, R. Panek, J. Y. Pascal, F. Saint-Laurent, et al., J. Nucl. Mater. 363-365, 484 (2007).

[41] S. K. Erents, A. V. Chankin, G. F. Matthews, and P. C. Stangeby, Plasma Phys. Control. Fusion 42, 905 (2000).

[42] S. K. Erents, R. A. Pitts, W. Fundamenski, J. P. Gunn, and G. F. Matthews, Plasma Phys. Control. Fusion 46,
1757 (2004).

[43] B. Labombard, S. Gangadhara, B. Lipschultz, and C. S. Pitcher, J. Nucl. Mater. 313-316, 995 (2003).

[44] Y. H. Xu, C. X. Yu, J. R. Luo, J. S. Mao, B. H. Liu, J. G. Li, B. N. Wan, and Y. X. Wan, Phys. Rev. Lett. 84, 3867 (2000).

[45] B. Goncalves, C. Hidalgo, M. A. Pedrosa, R. O. Orozco, E. Sanchez, and C. Silva, Phys. Rev. Lett. 96, 145001 (2006).

[46] V. Antoni, E. Spada, N. Vianello, M. Spolaore, R. Cavazzana, G. Serianni, and E. Martines, Plasma Phys. Control. Fusion 47, B13 (2005). 